Old Dominion University

ODU Digital Commons

Physics Theses \& Dissertations

Physics

Summer 2017

\title{
Test of New Readout Electronics for the BONuS12 Experiment
}

Mathieu Ehrhart

Old Dominion University

Follow this and additional works at: https://digitalcommons.odu.edu/physics_etds

Part of the Nuclear Commons

\section{Recommended Citation}

Ehrhart, Mathieu. "Test of New Readout Electronics for the BONuS12 Experiment" (2017). Master of Science (MS), Thesis, Physics, Old Dominion University, DOI: 10.25777/bwtc-tg68

https://digitalcommons.odu.edu/physics_etds/15

This Thesis is brought to you for free and open access by the Physics at ODU Digital Commons. It has been accepted for inclusion in Physics Theses \& Dissertations by an authorized administrator of ODU Digital Commons. For more information, please contact digitalcommons@odu.edu. 


\section{TEST OF NEW READOUT ELECTRONICS FOR THE BONUS12 EXPERIMENT}

by

Mathieu Ehrhart

B.S. May 2015, Old Dominion University

A Thesis Submitted to the Faculty of the Department of Physics Old Dominion University in Partial Fulfillment of the

Requirements for the Degree of

MASTER OF SCIENCE

PHYSICS

OLD DOMINION UNIVERSITY

August 2017

Approved by:

Stephen Bueltmann (Director)

Sebastian Kuhn (Member)

Gabriel Charles (Member) 


\title{
ABSTRACT \\ TEST OF NEW READOUT ELECTRONICS FOR THE BONUS12 EXPERIMENT
}

\author{
Mathieu Ehrhart \\ Old Dominion University, 2017 \\ Director: Dr. Stephen Bueltmann
}

For decades, electron-proton scattering experiments have been providing a large amount of data on the proton structure function. However, because of the instability of free neutrons, fewer experiments have been able to study the neutron structure function. The BONuS collaboration at Jefferson Laboratory addresses this challenge by scattering electrons off a deuterium target, using a RTPC capable of detecting the low-momentum spectator protons near the target. Events of electrons scattering on almost free neutrons are selected by constraining the spectator protons to very low momenta and very backward scattering angles. In 2005, BONuS successfully measured the neutron structure with scattering electrons of up to $5.3 \mathrm{GeV}$ energy. An extension of this measurement has been approved using the newly upgraded 12 GeV electron beam and CLAS12 (CEBAF Large Acceptance Spectrometer). For this new set of measurements, a new RTPC detector using GEM trackers is being developed to allow measurements of spectator protons with momenta as low as 70 $\mathrm{MeV} / c$.

The new RTPC will use a new readout electronic system, which is also used by other trackers in CLAS12. This thesis will present the first tests of this electronics using a previously built RTPC of similar design. 
Copyright, 2017, by Mathieu Ehrhart, All Rights Reserved. 


\section{ACKNOWLEDGEMENTS}

First and foremost I want to thank my advisor Dr. Stephen Bueltmann for his mentoring and his time. You are passionate about experimental nuclear physics, and contaminated me with the same passion. I will not forget our weekly conversations, eating some sugary treats, and talking about the "good old days".

I am grateful to the members of the ODU BONuS12 group for their good advice and collaboration: Dr. Gail Dodge, Nathan Dzbenski, David Payette, and Jiwan Poudel. But I am especially thankful for Dr. Sebastian Kuhn and Dr. Gabriel Charles, who are part of this thesis committee.

This challenging year was made enjoyable in large part due to the many friends and groups that became a part of my life.

Lastly, I would like to thank my family for all their encouragement and incredible support. I dedicate this thesis to you. 


\section{TABLE OF CONTENTS}

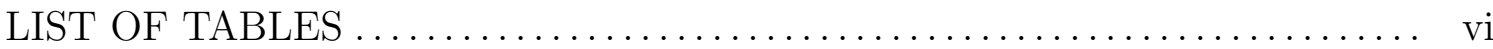

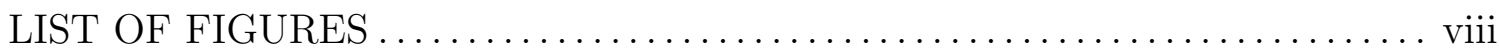

Chapter

1. PHYSICS BACKGROUND $\ldots \ldots \ldots \ldots \ldots \ldots \ldots \ldots \ldots \ldots \ldots \ldots \ldots \ldots \ldots \ldots \ldots \ldots \ldots \ldots \ldots$

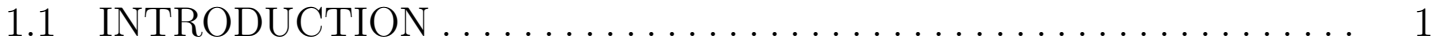

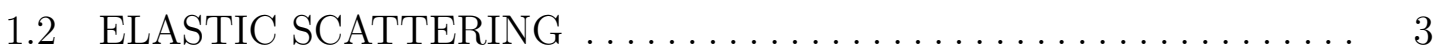

1.3 DEEP INELASTIC SCATTERING $\ldots \ldots \ldots \ldots \ldots \ldots \ldots \ldots \ldots$

1.4 THE NEUTRON STRUCTURE FUNCTIONS . . . . . . . . . . . . 9

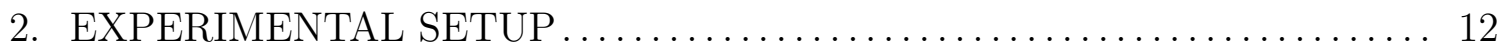

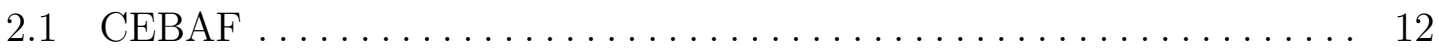

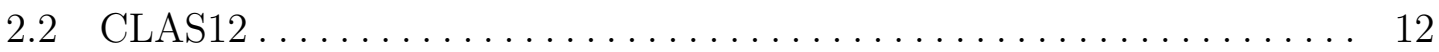

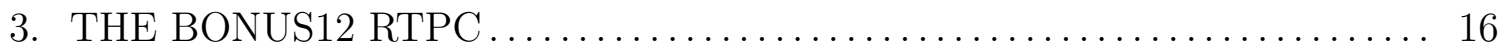

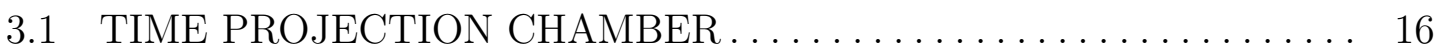

3.2 GAS ELECTRON MULTIPLIER . . . . . . . . . . . . . . . . . . 17

3.3 RADIAL TIME PROJECTION CHAMBERS . . . . . . . . . . . . . 19

3.4 PREVIOUS READOUT ELECTRONICS $\ldots \ldots \ldots \ldots \ldots \ldots \ldots \ldots \ldots . \ldots \ldots$

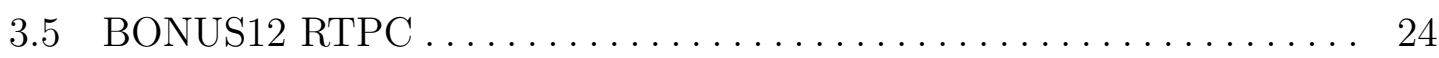

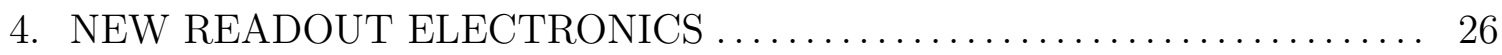

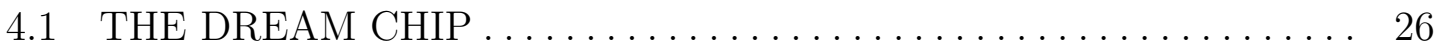

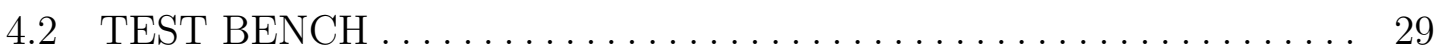

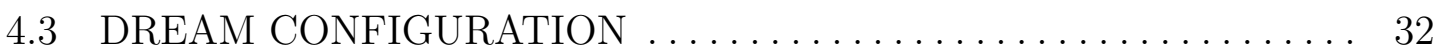

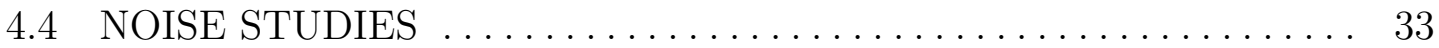

4.5 DATA WITH A SOURCE $\ldots \ldots \ldots \ldots \ldots \ldots \ldots \ldots \ldots \ldots \ldots \ldots \ldots$

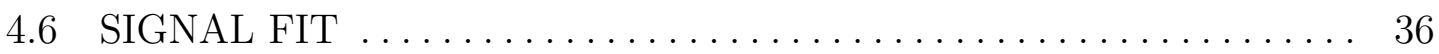

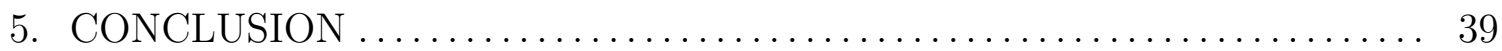

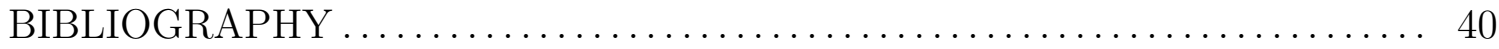

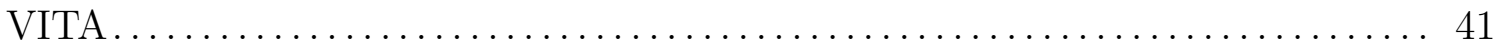




\section{LIST OF TABLES}

Table

1. Key parameters of the CEBAF $12 \mathrm{GeV}$ Upgrade ................. 13

2. Design characteristics of the CLAS12 detector $\ldots \ldots \ldots \ldots \ldots \ldots \ldots \ldots$

3. Summary of the DREAM requirements $[14] \ldots \ldots \ldots \ldots \ldots \ldots$ 


\section{LIST OF FIGURES}

Figure

Page

1. Particles of the Standard Model of Particle Physics $\ldots . \ldots \ldots \ldots \ldots \ldots \ldots$

2. Diagram of an electron elastically scattering off a nucleus $\ldots \ldots \ldots \ldots \ldots$

3. Feynman diagram of elastic scattering $\ldots \ldots \ldots \ldots \ldots \ldots \ldots \ldots \ldots$

4. Diagram of an electron scattering off a proton in Deep Inelastic Scattering 5

5. Spectrum of scattered electrons from electron-proton scattering at an electron energy of $E=4.9 \mathrm{GeV}$ and a scattering angle of $\theta=10^{\circ}[3] \ldots \ldots$

6. $F_{2}^{e p}\left(x, Q^{2}\right)$ versus $Q^{2}$ showing the independence of the structure functions

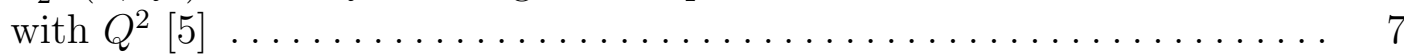

7. Ratio $\frac{2 x F_{1}}{F_{2}}$ demonstrating its approximate unity $[5] \ldots \ldots \ldots \ldots \ldots \ldots$

8. Diagram of electron deep inelastic scattering off a neutron with spectator

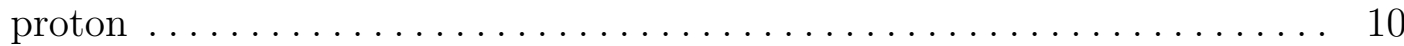

9. Ratio $\frac{F_{2}^{e n}}{F_{2}^{e p}}$ for three different minimum values of $W$. The data are compared to a phenomenological prediction (yellow band) [8] and the systematic uncertainties are shown in red $\ldots \ldots \ldots \ldots \ldots \ldots \ldots \ldots \ldots \ldots \ldots$

10. Jefferson Lab $12 \mathrm{GeV}$ upgrade...................... 12

11. Split view of the Forward Detector . . . . . . . . . . . . . . . . . . . 14

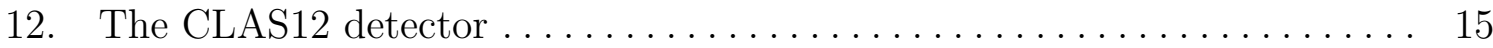

13. Cross-section of the DELPHI TPC $\ldots \ldots \ldots \ldots \ldots \ldots \ldots \ldots \ldots \ldots \ldots \ldots$

14. Picture of a GEM under an electron microscope $\ldots \ldots \ldots \ldots \ldots \ldots \ldots \ldots$

15. Electric field near the holes of a GEM . . . . . . . . . . . . . . . . . . . 18

16. Triple GEM design $\ldots \ldots \ldots \ldots \ldots \ldots \ldots \ldots \ldots \ldots \ldots \ldots \ldots \ldots \ldots \ldots$

17. Cross section of the eg6 RTPC (along the beamline) . . . . . . . . . 20

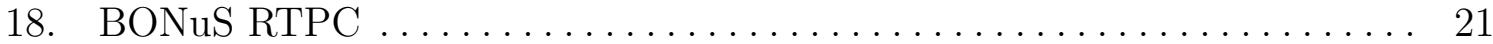


19. Reconstructed track of a spectator proton with the BONuS RTPC . . . . 22

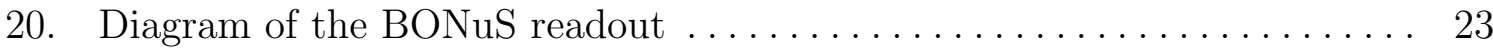

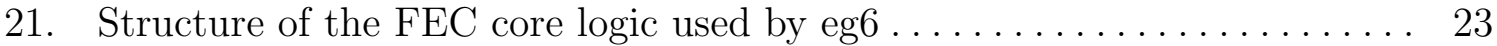

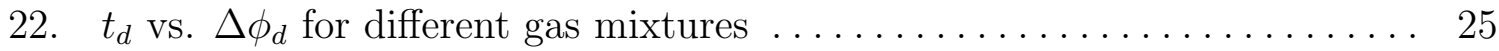

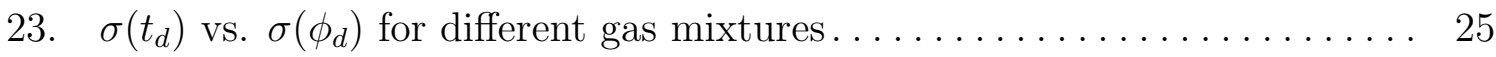

24. Block diagram of the DREAM chip $[14] \ldots \ldots \ldots \ldots \ldots \ldots \ldots \ldots \ldots$

25. DREAM electronics readout architecture $[15] \ldots \ldots \ldots \ldots \ldots \ldots \ldots \ldots$

26. Modular structure of the FEU core logic $[15] \ldots \ldots \ldots \ldots \ldots \ldots \ldots \ldots$

27. Front and back pictures of the ODU test bench $\ldots \ldots \ldots \ldots \ldots \ldots \ldots$

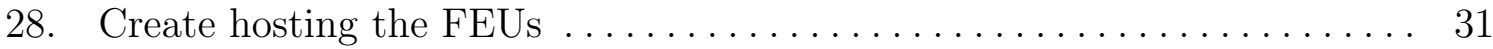

29. 3D drawing of the ODU RTPC adaptation card $\ldots \ldots \ldots \ldots \ldots \ldots \ldots \ldots$

30. Noise levels for the channels connected to the RTPC . . . . . . . . . 33

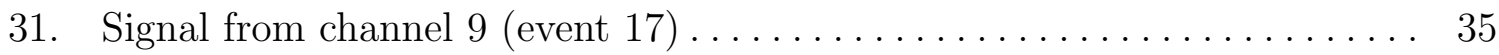

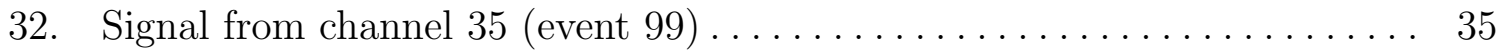

33. Double Gaussian fit $[$ Ch 9 - Evt 17$] \ldots \ldots \ldots \ldots \ldots \ldots \ldots \ldots \ldots \ldots \ldots \ldots$

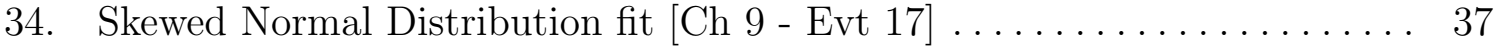

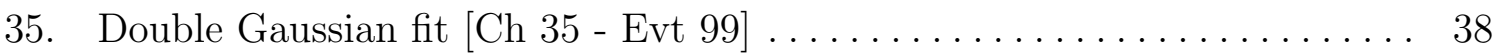

36. Skewed Normal Distribution fit $[$ Ch 35 - Evt 99] ............. 38 


\section{CHAPTER 1}

\section{PHYSICS BACKGROUND}

\subsection{INTRODUCTION}

From Ancient Greece to our days, understanding what makes us and the world around us has been a source of questioning for philosophers, early scientists and physicists. From the four classical elements of matter - earth, air, fire, water - to the quarks, the search for the fundamental building blocks of matter took over two millennia, and is far from being over. Strong and rapid developments occurred in the last century, allowing us to get closer to the ultimate answer to this puzzle.

Our current model, the Standard Model, defines twelve fundamental particles (and their anti-particle conjugates), summarized in Fig. 1.

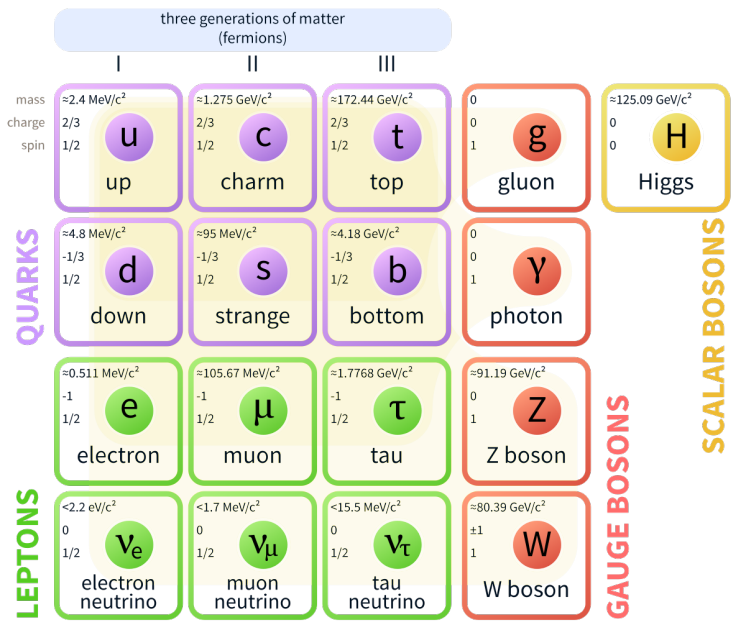

FIG. 1: Particles of the Standard Model of Particle Physics

While the Standard Model explains a large number of observables in Particle Physics, this thesis will focus on the quarks and the gluons. Quarks can be combined in pairs to create mesons, or in groups of three quarks to form baryons. Despite a large number of hadron combinations possible, only two of them make up the nucleons inside the nucleus of atoms: the proton (uud) and the neutron $(u d d)$. 
The quarks are held together by the strong force, which is one of the four fundamental forces together with gravity, the electromagnetic force and the weak force. The gluons are the vector gauge bosons (the exchange particles) that carry the strong force. The theory explaining the quark-gluon interaction is called quantum chromodynamics (QCD).

In QCD (SU(3)), each quark has one of the three possible "color" charges: red $(r)$, blue $(b)$ and green $(g)$. This color charge is analogous to the electric charge in quantum electrodynamics $(\mathrm{U}(1))$. Since a massless gluon carries one color charge and one anti-color charge, they can exist in nine states and can also interact with each other. Since QCD is a non-Abelian gauge theory with symmetry group $\mathrm{SU}(3)$, the gluons can be arranged into a "color singlet",

$$
\frac{1}{\sqrt{3}}(r \bar{r}+b \bar{b}+g \bar{g})
$$

which does not correspond to a physical gluon, and "color octet",

$$
\begin{array}{cl}
\frac{1}{\sqrt{2}}(r \bar{b}+b \bar{r}) & -\frac{i}{\sqrt{2}}(r \bar{b}-b \bar{r}) \\
\frac{1}{\sqrt{2}}(r \bar{r}-b \bar{b}) & \frac{1}{\sqrt{2}}(r \bar{g}+g \bar{r}) \\
-\frac{i}{\sqrt{2}}(r \bar{g}-g \bar{r}) & \frac{1}{\sqrt{2}}(b \bar{g}+g \bar{b}) \\
-\frac{i}{\sqrt{2}}(b \bar{g}-g \bar{b}) & \frac{1}{\sqrt{6}}(r \bar{r}+b \bar{b}-2 g \bar{g})
\end{array}
$$

One can ask how quarks and gluons are distributed inside the nucleon. In order to access this information, high energy leptons (e.g. electrons, muons, neutrinos, ...) are used to probe the nucleons. 


\subsection{ELASTIC SCATTERING}

In order to understand the process of high energy electron scattering, one must go back to its origins. In the 1950s, experiments took place at Stanford that showed that the nucleons are not point-like particles, but have a finite size of about $1 \mathrm{fm}$ [1].

Dealing with high energy (relativistic) particles, four-momentum vectors have to be used for a particle's momentum:

$$
p=\left(p_{0}, p_{1}, p_{2}, p_{3}\right)=(E / c, \mathbf{p})
$$

The first type of experiment conducted to probe the structure of the nucleon using electrons were elastic scattering experiments. This process gives access to the electromagnetic form factors of the nucleons. In an elastic scattering experiment, an electron is scattered off a target - e.g. proton - leaving it intact and both objects are recoiled at different angles with respected to the incident electron path, see Fig. 2.
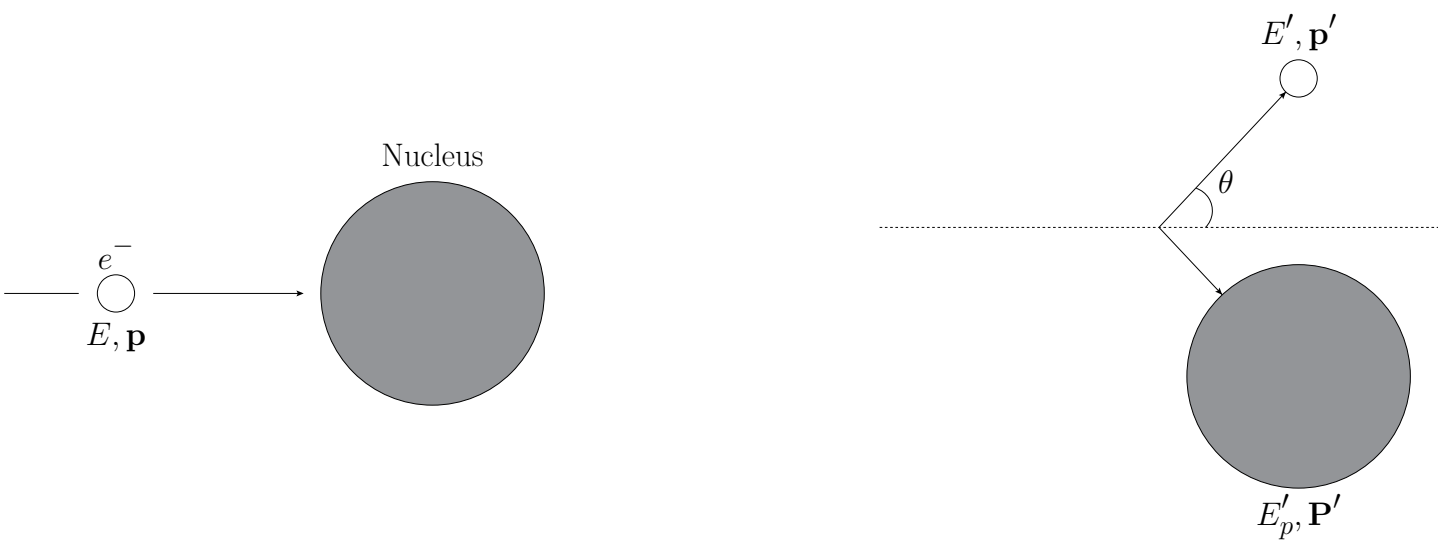

FIG. 2: Diagram of an electron elastically scattering off a nucleus

Fig. 3 shows the Feynman diagram of the process. In order to access the form factors, an important quantity needs to be defined, the virtuality of the photon $\left(Q^{2}\right)$ [2]:

$$
Q^{2} \equiv-q^{2}=4 E E^{\prime} \sin ^{2} \frac{\theta}{2}
$$

where $q=\left(\left(E-E^{\prime}\right) / c, \mathbf{q}\right)$ is the 4-momentum transferred to the proton. 


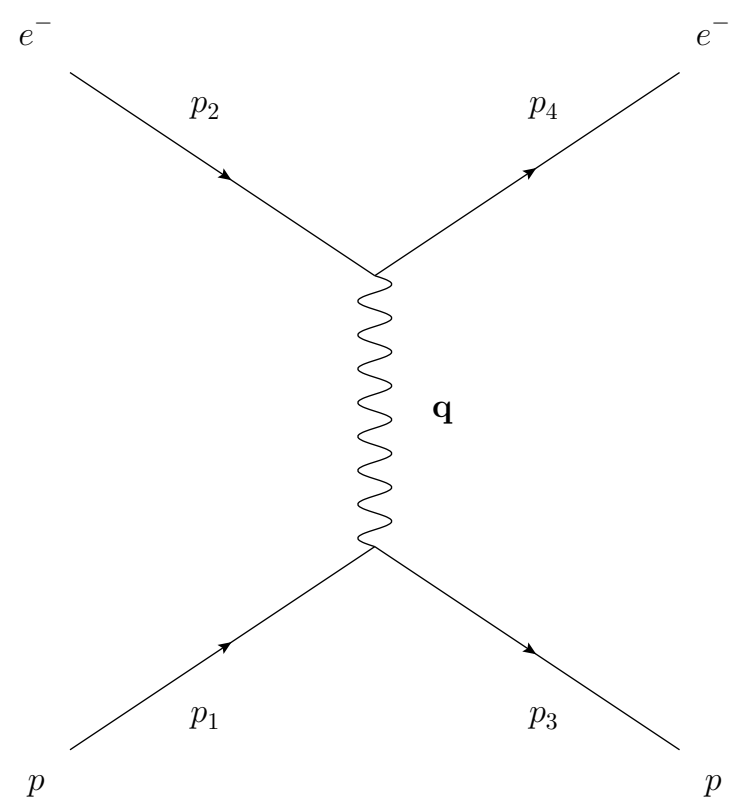

FIG. 3: Feynman diagram of elastic scattering

Detectors close to the interaction point will measure the properties of the particles coming out of the scattering process (trajectories, type of particles, energies, ...) allowing the calculation of cross sections and giving access to the form factors $G_{E}\left(Q^{2}\right)$ and $G_{M}\left(Q^{2}\right)$.

The electric $G_{E}\left(Q^{2}\right)$ and magnetic $G_{M}\left(Q^{2}\right)$ form factors are a combination of the Dirac and Pauli form factors $F_{1}\left(Q^{2}\right)$ and $F_{2}\left(Q^{2}\right)$ (Sachs parametrization):

$$
\begin{aligned}
G_{E}\left(Q^{2}\right) & =F_{1}\left(Q^{2}\right)+\frac{Q^{2}}{4 M^{2}} F_{2}\left(Q^{2}\right) \\
G_{M}\left(Q^{2}\right) & =F_{1}\left(Q^{2}\right)+F_{2}\left(Q^{2}\right)
\end{aligned}
$$

The form factors can be extracted from cross section measurements using the Rosenbluth formula [2]:

$$
\left(\frac{d \sigma}{d \Omega}\right)_{\exp }=\left(\frac{d \sigma}{d \Omega}\right)_{\text {Mott }} \frac{E^{\prime}}{E}\left(\frac{G_{E}^{2}\left(Q^{2}\right)+\tau G_{M}^{2}\left(Q^{2}\right)}{1+\tau}+2 \tau G_{M}^{2}\left(Q^{2}\right) \tan ^{2} \frac{\theta}{2}\right)
$$


where:

$$
\begin{aligned}
\left(\frac{d \sigma}{d \Omega}\right)_{\text {Mott }} & =\frac{\alpha^{2}}{4 E^{2} \sin ^{4} \frac{\theta}{2}} \cos ^{2} \frac{\theta}{2} \\
\tau & =\frac{Q^{2}}{4 M^{2}} \\
\alpha & =\frac{e}{4 \pi} \approx \frac{1}{137}
\end{aligned}
$$

$\frac{d \sigma}{d \Omega}$ represents the number of particles scattered into the solid angle $(d \Omega)$ per unit time per target particle as a function of the incident flux.

The form factors can be thought of as the Fourier transforms of the charge and magnetization current densities inside the nucleon.

\subsection{DEEP INELASTIC SCATTERING}

The first Deep Inelastic Scattering (DIS) occurred at Stanford in the 1960s and showed the quark substructure of nucleons. In DIS, the wavelength $\lambda$ of the virtual photon is smaller than the size of the nucleon $(\sim 1 \mathrm{fm})$, allowing to probe the constituents of the nucleons - the partons. Since $\lambda$ is proportional to $1 / \sqrt{Q^{2}}$, deep inelastic scattering requires a high photon virtuality, therefore a high electron energy. Fig. 4 is the diagram of the reaction:

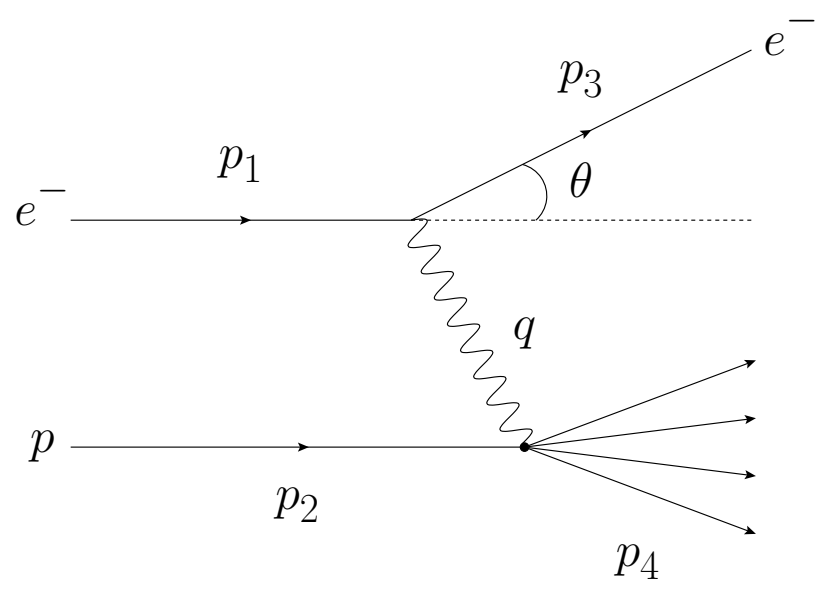

FIG. 4: Diagram of an electron scattering off a proton in Deep Inelastic Scattering 
In DIS, the probed nucleon breaks up, creating a complex hadronic final state. A new variable defines this process and is called the invariant mass, $W^{2}$ :

$$
W^{2}=M^{2}+2 M \nu-4 E E^{\prime} \sin ^{2} \frac{\theta}{2}
$$

where $\nu=E-E^{\prime}$.

For $W^{2}=M \approx 1 \mathrm{GeV} / \mathrm{c}^{2}$, the scattering will be elastic. Up to $W^{2} \approx 2 \mathrm{GeV} / \mathrm{c}^{2}$, nucleon resonances are observed. Finally, $W^{2} \gtrsim 2 \mathrm{GeV} / \mathrm{c}^{2}$ is the deep inelastic regime (see Fig. 5).

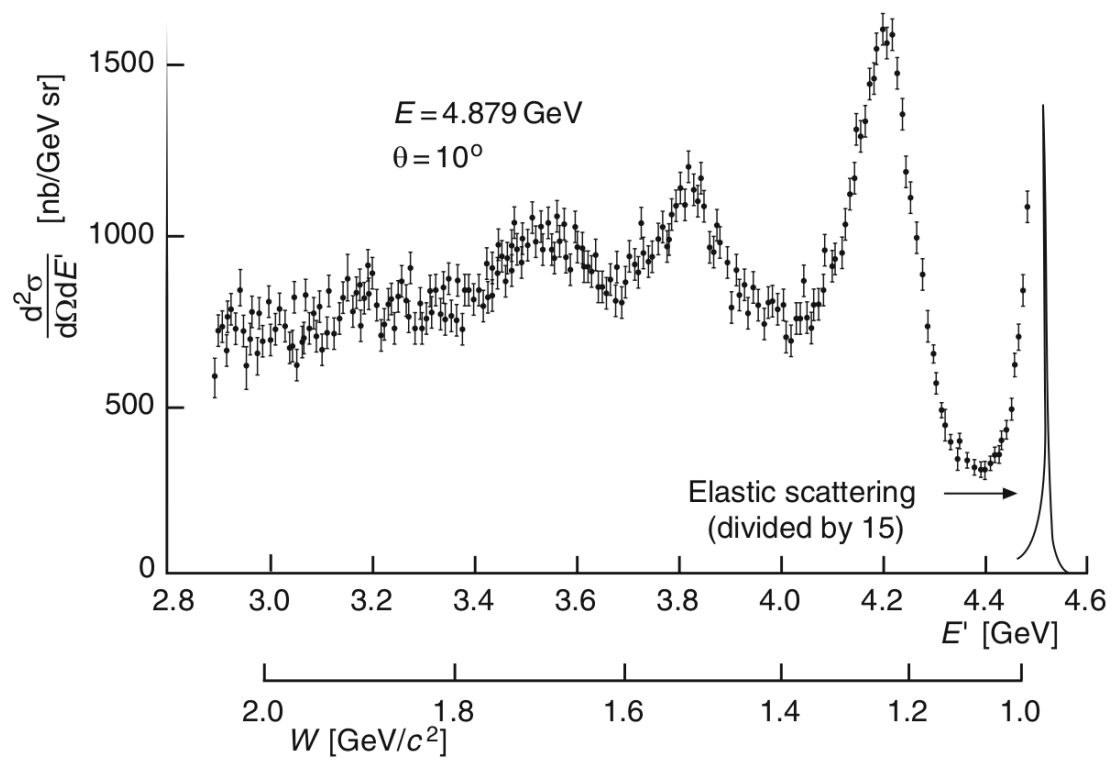

FIG. 5: Spectrum of scattered electrons from electron-proton scattering at an electron energy of $E=4.9 \mathrm{GeV}$ and a scattering angle of $\theta=10^{\circ}[3]$

It is also convenient to introduce two new scaling variable $x$ (Bjorken scaling) [4] and $y$ :

$$
\begin{aligned}
& x=\frac{Q^{2}}{2 p_{2} q} \\
& y=\frac{p_{2} q}{p_{1} p_{2}}
\end{aligned}
$$


The Rosenbluth formula for elastic scattering can be rewritten using this scaling and $Q^{2}$ :

$$
\frac{d \sigma}{d Q^{2}}=\frac{4 \pi \alpha^{2}}{Q^{4}}\left[\frac{G_{E}^{2}\left(Q^{2}\right)+\tau G_{M}^{2}\left(Q^{2}\right)}{1+\tau}\left(1-y-\frac{M^{2} y^{2}}{Q^{2}}\right)+\frac{1}{2} y^{2} G_{M}^{2}\right]
$$

Introducing $f_{2}\left(Q^{2}\right)=\frac{G_{E}^{2}\left(Q^{2}\right)+\tau G_{M}^{2}\left(Q^{2}\right)}{1+\tau}$ and $f_{1}\left(Q^{2}\right)=G_{M}^{2}$, equation 10 becomes:

$$
\frac{d \sigma}{d Q^{2}}=\frac{4 \pi \alpha^{2}}{Q^{4}}\left[f_{2}\left(Q^{2}\right)\left(1-y-\frac{M^{2} y^{2}}{Q^{2}}\right)+\frac{1}{2} y^{2} f_{1}\left(Q^{2}\right)\right]
$$

Deep Inelastic Scattering allows the extraction of the nucleon structure function $F_{1}\left(x, Q^{2}\right)$ and $F_{2}\left(x, Q^{2}\right)$ :

$$
\frac{d \sigma}{d x d Q^{2}}=\frac{4 \pi \alpha^{2}}{Q^{4}}\left[\frac{F_{2}\left(x, Q^{2}\right)}{x}\left(1-y-\frac{M^{2} y^{2}}{Q^{2}}\right)+y^{2} F_{1}\left(x, Q^{2}\right)\right]
$$

The first experiments, performed at SLAC in the 1960s, showed that $F_{1}\left(x, Q^{2}\right)$ and $F_{2}\left(x, Q^{2}\right)$ are approximately independent of $Q^{2}$, leading to the following asymptotic behavior at large $Q^{2}: F_{1}\left(x, Q^{2}\right) \rightarrow F_{1}(x)$ and $F_{2}\left(x, Q^{2}\right) \rightarrow F_{2}(x)$ (see Fig. 6).

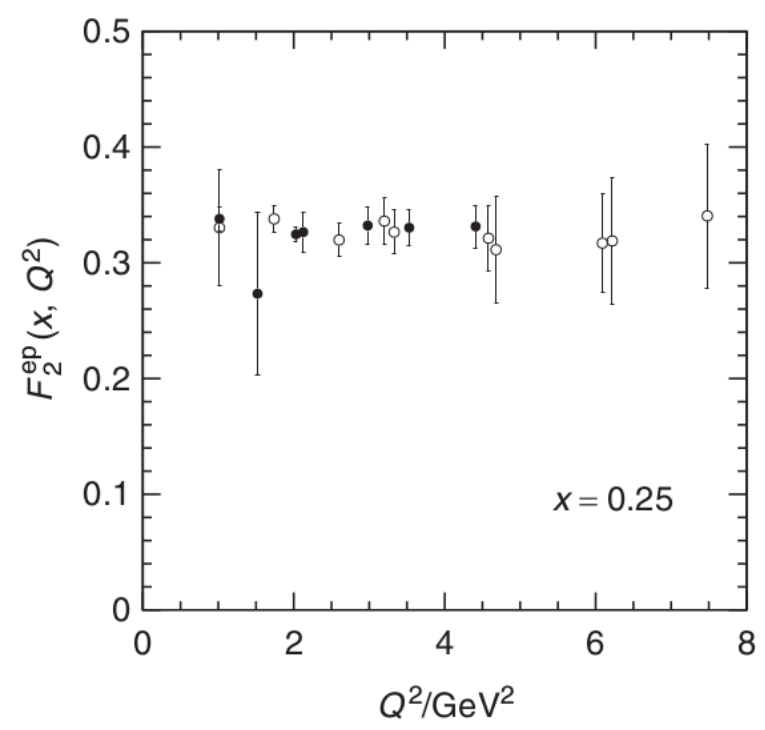

FIG. 6: $F_{2}^{e p}\left(x, Q^{2}\right)$ versus $Q^{2}$ showing the independence of the structure functions with $Q^{2}[5]$ 
It was also found that the two structure functions are not independent of each other, but rather satisfy the Callan-Gross relation $F_{2}(x)=2 x F_{1}(x)$ in the same limit (see Fig. 7).

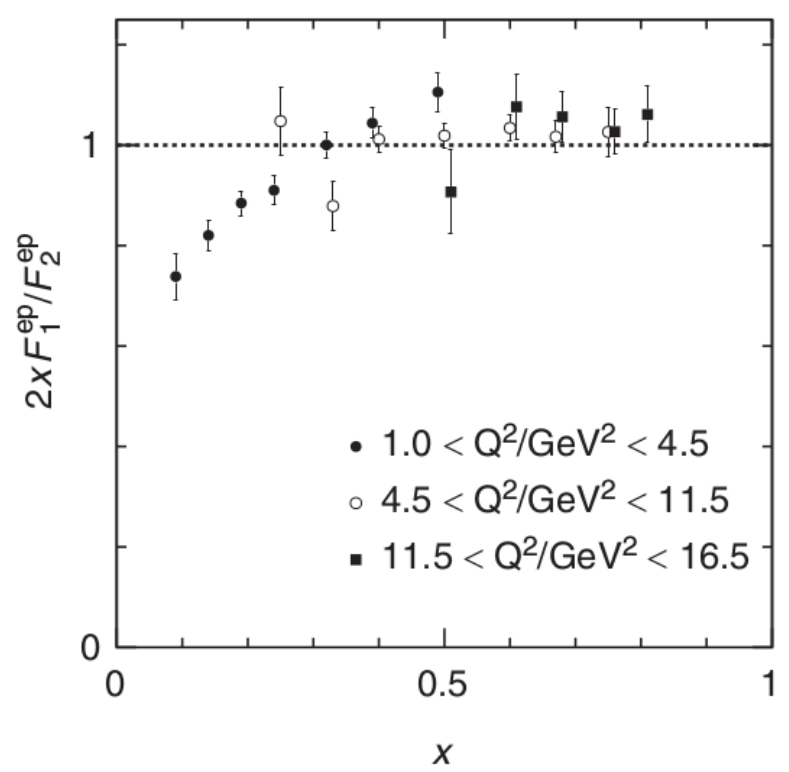

FIG. 7: Ratio $\frac{2 x F_{1}}{F_{2}}$ demonstrating its approximate unity [5]

The structure functions are used to calculate the parton distribution functions, which are related to $F_{2}(x)$ by the following relation:

$$
F_{2}^{e}(x)=x \cdot \sum_{q} e_{q} f_{q}(x)
$$

where $e_{q}$ is the charge of the quark and $f_{q}(x)$ is the parton distribution function of the quark.

In the case of electron-proton $(u u d)$ scattering, the relation becomes:

$$
F_{2}^{e p}(x)=x\left(\frac{4}{9} u^{p}(x)+\frac{1}{9} d^{p}(x)+\frac{4}{9} \bar{u}^{p}(x)+\frac{4}{9} \bar{d}^{p}(x)\right)
$$

Similarly, for electron-neutron $(d d u)$ scattering:

$$
F_{2}^{e p}(x)=x\left(\frac{4}{9} u^{n}(x)+\frac{1}{9} d^{n}(x)+\frac{4}{9} \bar{u}^{n}(x)+\frac{4}{9} \bar{d}^{n}(x)\right)
$$


From isospin symmetry, if the following assumption is made:

$$
\begin{array}{ll}
d^{n}(x)=u^{p}(x) \equiv u(x) & u^{n}(x)=d^{p}(x) \equiv d(x) \\
\bar{d}^{n}(x)=\bar{u}^{p}(x) \equiv \bar{u}(x) & \bar{u}^{n}(x)=\bar{d}^{p}(x) \equiv \bar{d}(x)
\end{array}
$$

equations (14) and (15) become:

$$
\begin{aligned}
& F_{2}^{e p}(x)=x\left(\frac{4}{9} u(x)+\frac{1}{9} d(x)+\frac{4}{9} \bar{u}(x)+\frac{4}{9} \bar{d}(x)\right)=2 x F_{1}^{e p}(x) \\
& F_{2}^{e n}(x)=x\left(\frac{4}{9} d(x)+\frac{1}{9} u(x)+\frac{4}{9} \bar{d}(x)+\frac{4}{9} \bar{u}(x)\right)=2 x F_{1}^{e n}(x)
\end{aligned}
$$

\subsection{THE NEUTRON STRUCTURE FUNCTIONS}

In the proton, since the photon will have a higher coupling strength with the $u$ quark, its parton distribution function $u(x)$ can be accurately measured. However, in order to accurately determine the $d(x)$ function, neutron data are needed to extract the $d / u$ ratio:

$$
\frac{d(x)}{u(x)} \approx \frac{4 F_{2}^{e n}(x) / F_{2}^{e p}(x)-1}{4-F_{2}^{e n}(x) / F_{2}^{e p}(x)}, \quad \text { for } x \gtrsim 0.5
$$

The proton structure functions have been extensively studied for the past 50 years, due to the stability of protons. A hydrogen target is the perfect way to study the proton, it has a practically infinite lifetime and can be produced at high density. However, data for the neutron structure functions are a bit more difficult to obtain. Free neutron targets are not easy to produce and have a half-life of about 15 minutes, making any experiments challenging.

The stucture function $F_{2}^{e n}$ can be extracted from electron scattering off deuterium. The binding energy of the deuteron, deuterium's nucleus - composed of a neutron and a proton - is $-2.2 \mathrm{MeV}$ or $0.1 \%$ of its mass, making it the perfect candidate to study the neutron.

In order to select a electron-neutron event, the recoil proton $-p_{s}$ - needs to be detected or "tagged", in the reaction $e^{\prime}+D \rightarrow e^{\prime}+p_{s}+X$, shown in Fig. 8: 


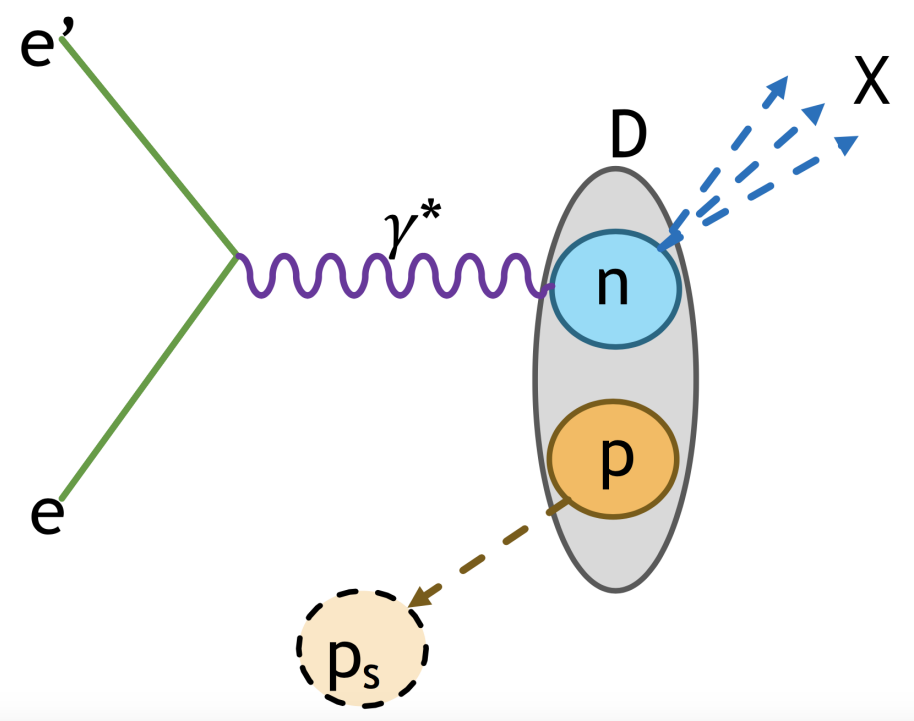

FIG. 8: Diagram of electron deep inelastic scattering off a neutron with spectator proton

This tagging method allows the selection of events for which the neutron was weakly bound to the proton in the scattering process, had a low-momentum and also minimizing the final state interactions (FSI) with the spectator proton. This can be achieved by carefully selecting events where $p_{s}<100 \mathrm{MeV} / c$ and by limiting the spectator angle $\theta_{p q}>100^{\circ}[6]$.

In 2005, the "Barely Off-Shell Nucleon Structure" experiment, or BONuS experiment, measured the neutron structure functions with the CLAS spectrometer at Jefferson Lab, for $Q^{2}$ between 0.7 and $5 \mathrm{GeV}^{2} / c^{2}$, with invariant mass $W$ between 1 and $2.7 \mathrm{GeV} / c^{2}$, and Bjorken $x$ between 0.25 and 0.6. The BONuS experiment required the use of a new detector, a Radial Time Projection Chamber (RTPC), which was specifically designed for the detection of the spectator protons down to $70 \mathrm{MeV} / \mathrm{c}$. The results of the BONuS experiment are summarized in Fig. 9 [7]. 


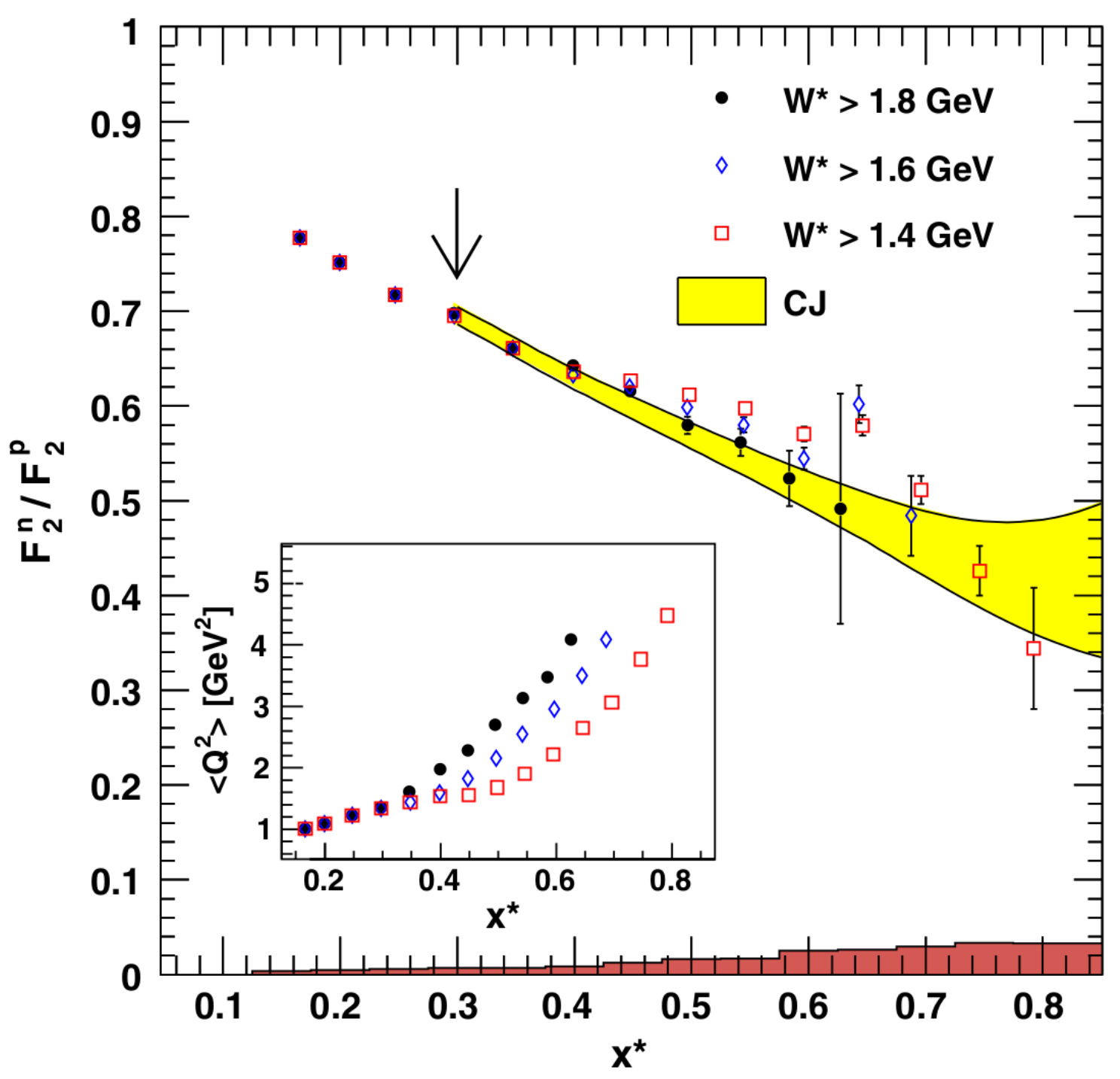

FIG. 9: Ratio $\frac{F_{2}^{e n}}{F_{2}^{e p}}$ for three different minimum values of $W$. The data are compared to a phenomenological prediction (yellow band) [8] and the systematic uncertainties are shown in red 


\section{CHAPTER 2}

\section{EXPERIMENTAL SETUP}

\subsection{CEBAF}

The Continuous Electron Beam Accelerator Facility (CEBAF) is the main research facility at the Thomas Jefferson National Accelerator Facility or Jefferson Lab, located in Newport News, Virginia. It is composed of two Linear Accelerators (LINAC) connected on both ends by recirculating arcs made of bending and focusing magnets (Fig. 10).

In the newly upgraded version of the accelerator, the extracted beam energy can reach up to $12 \mathrm{GeV}$ in Hall $\mathrm{D}$, and $11 \mathrm{GeV}$ in the three other halls (A,B and C). Jefferson Lab was the first laboratory to use Superconducting Radio Frequency (SFR) technology on a large scale. Since SRF accelerating cavities require low temperatures to accelerate electrons, two central helium liquefiers cool down helium to about $2 \mathrm{~K}$. The electron beam can travel up to fives times around the "racetrack" to reach the highest beam energy (Table 1).

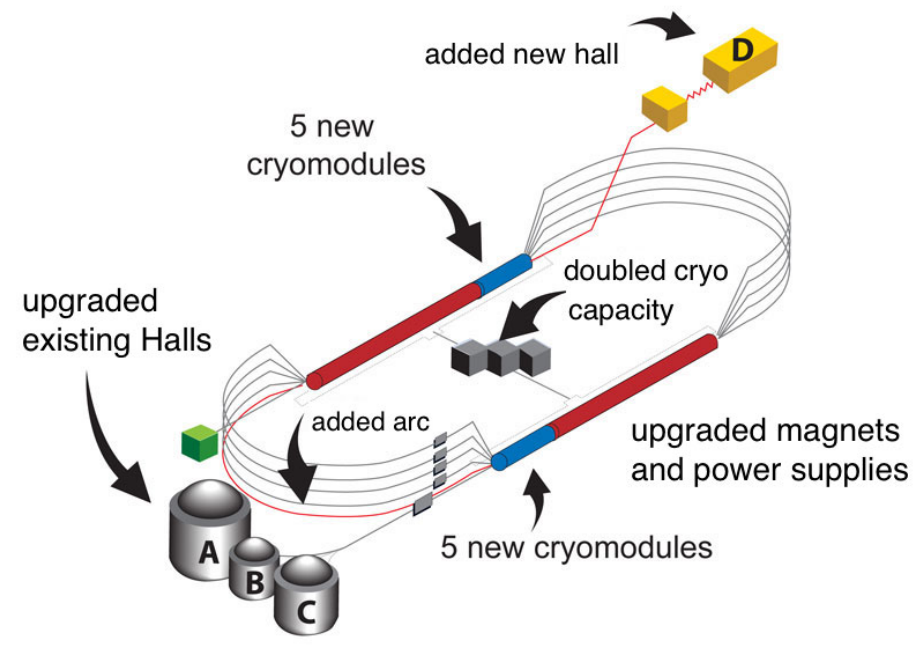

FIG. 10: Jefferson Lab 12 GeV upgrade 
TABLE 1: Key parameters of the CEBAF 12 GeV Upgrade

\begin{tabular}{ll}
\hline \hline Parameter & Specification \\
\hline Max. energy gain per pass & $2.2 \mathrm{GeV}$ \\
Number of passes for Halls A, B, C & 5 \\
Number of passes for Hall D & 5.5 (add a tenth arc) \\
Max. energy to hall A, B, C & $11.0 \mathrm{GeV}$ \\
Max. energy to Hall D & $12.0 \mathrm{GeV}$ (for $9 \mathrm{GeV}$ photons) \\
Max. summed current to Halls A, C & $85 \mu \mathrm{A}$ \\
Max. summed current to Hall B, D & $5 \mu \mathrm{A}$ \\
\hline \hline
\end{tabular}

\subsection{CLAS12}

CLAS12, the "CEBAF Large Acceptance Spectrometer" is located in Hall B and is currently under construction. The new detector will have two different parts capable of handling a luminosity of up to $\mathcal{L}=10^{35} \mathrm{~cm}^{-2} \mathrm{~s}^{-1}$ : a Forward Detector and a Central Detector. At the heart of the Forward Detector is a torus magnet composed of six superconducting coils symmetrical arranged around the beam axis, producing a peak field of $3.5 \mathrm{~T}$. A number of baseline detectors are installed in between the coils of the torus and beyond (Fig. 11):

- the Drift Chambers (DC) will measure the momentum of charged particles being bent by the torus' magnetic field. They are arranged in three different regions throughout the Forward Detector with each region segmented into six sectors organized between the coils of the torus. The chambers will be filled with a mixture of $90 \%$ argon and $10 \% \mathrm{CO}_{2}$, with each cell expected to have a spatial resolution of about $250-350 \mu \mathrm{m}$

- two Cherenkov counters will also be available. The High Threshold Cherenkov Counter (HTCC) will be used to generated a fast trigger signal and reject background. The Low Threshold Cherenkov Counter (LTCC), located downstream of the target, will be used for kaon/pion separation from 3.5 to $9 \mathrm{GeV} / c$

- the Electromagnetic Calorimeter (EC) will be capable of identifying electrons and neutral particles and will be included in the trigger. The EC was already 
used in the previous CLAS detector. It is a sandwich of three planes of scintillator bars oriented at different angles with lead sheets in between. A pre-shower calorimeter (PCAL) will improve its spatial resolution by a factor of 2.5 , as well as the separation of two photons for momentum up to $10 \mathrm{GeV} / c$.

- the Forward Time-of-Flight (FTOF) will measure the time-of-flight of charged particles and will separate pions/kaons up to $2.8 \mathrm{GeV}$, kaons/protons up to $4.8 \mathrm{GeV}$, and pions/protons up to $5.4 \mathrm{GeV}$.

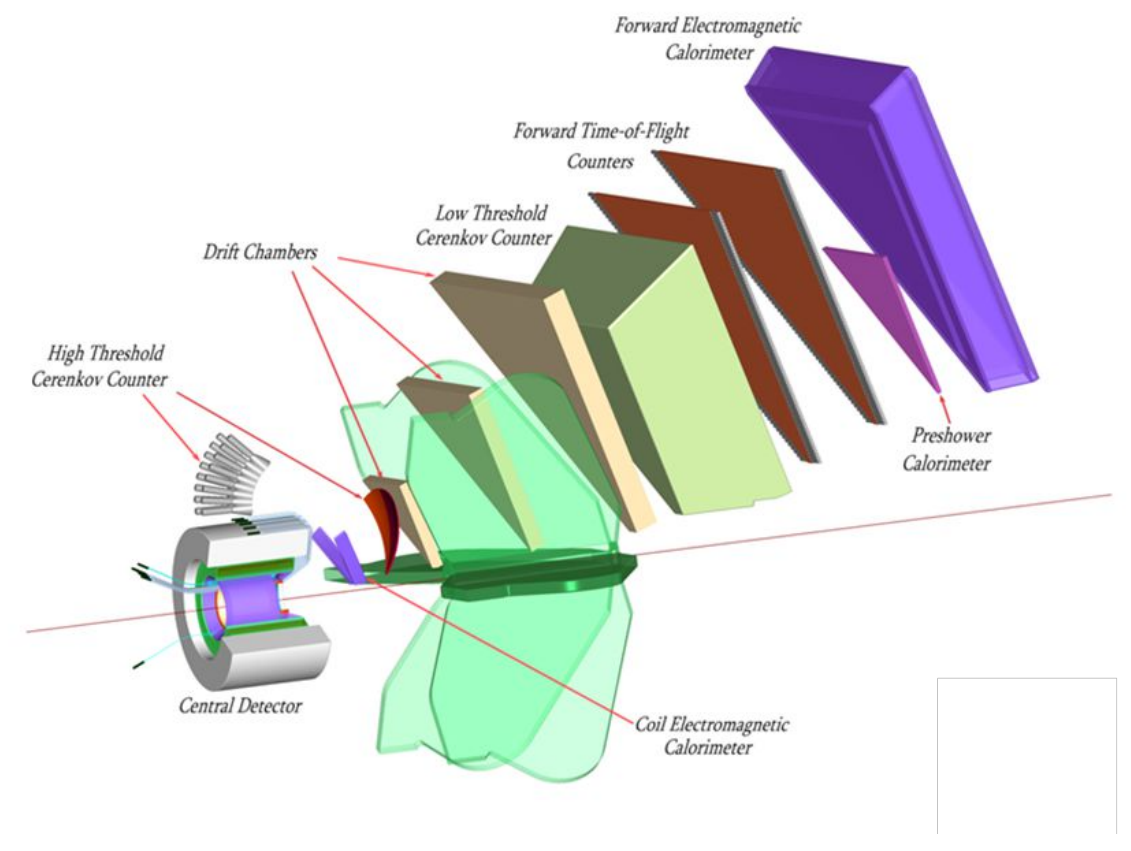

FIG. 11: Split view of the Forward Detector

The Central Detector is composed of three detectors, the Silicon Vertex Tracker (SVT) and the Micromegas Vertex Tacker (MVT) providing tracking information. The particle identification of lower energy particles (up to $1.2 \mathrm{GeV} / c$ for $\pi / p$ and up to $0.65 \mathrm{GeV} / c$ for $\pi / K$ ) is performed by the Central Time-of-Flight (CTOF). The Central Neutron Detector (CND) - a neutron Time-of-Flight — will detect neutrons and is the outmost layer of the Central Detector. They surround the target area and are located inside the superconducting solenoid magnet with a maximum central magnetic field of $5 \mathrm{~T}$.

A summary of the characteristics of CLAS12 can be found in Table 2, as well as an overall drawing of the detector (Fig. 12). 
TABLE 2: Design characteristics of the CLAS12 detector

\begin{tabular}{lcc}
\hline \hline Parameters & Forward Detector & Central Detector \\
\hline Charged particles: & $5^{\circ}$ to $35^{\circ}$ & $35^{\circ}$ to $125^{\circ}$ \\
polar angular range $(\theta)$ & $<1 \mathrm{mr}$ & $<10 \mathrm{mr}-20 \mathrm{mr}$ \\
polar angular resolution $(\delta \theta)$ & $<4 \mathrm{mr}$ & $<5 \mathrm{mr}$ \\
azimuthal angular resolution $(\delta \phi)$ & $<1 \%$ @ $5 \mathrm{GeV} / c$ & $<5 \% @ 1.5 \mathrm{GeV} / c$ \\
momentum resolution & & - \\
Neutral particles: & $5^{\circ}$ to $40^{\circ}$ & - \\
polar angular range $(\theta)$ & $<4 \mathrm{mr}$ & - \\
polar angular resolution $(\delta \theta)$ & $0.1 / \sqrt{E}$ & - \\
energy resolution $(\sigma E)$ & & $<1.25 \mathrm{GeV} / c$ \\
Particle identification: & full range & $<.65 \mathrm{GeV} / c$ \\
$e / \pi$ & full range & \\
$\pi / p$ & full range & $<\mathrm{GeV} / c$ \\
$K / \pi$ & $<4 \mathrm{GeV} / c$ & $<$ \\
$K / p$ &
\end{tabular}

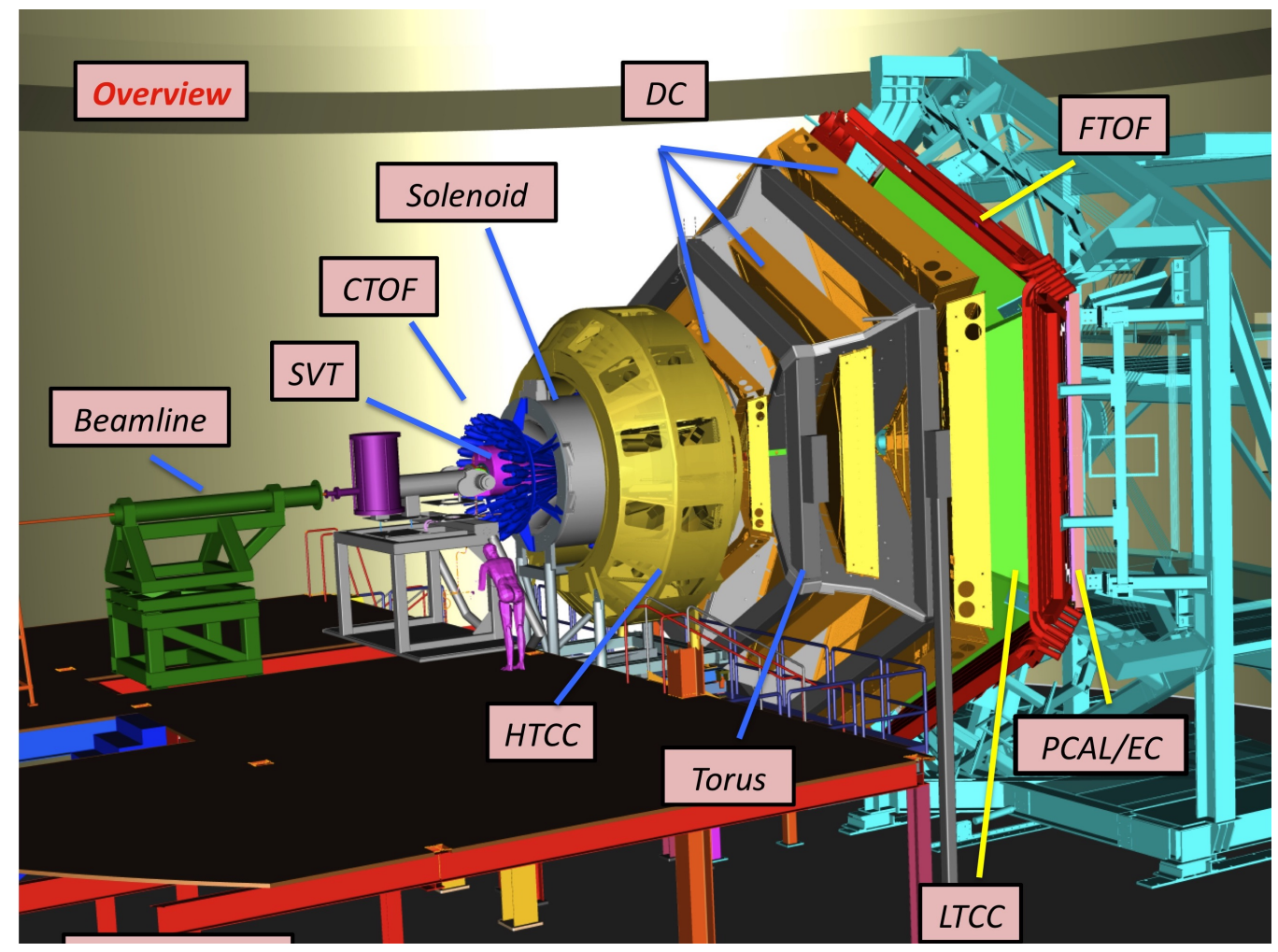

FIG. 12: The CLAS12 detector 


\section{CHAPTER 3}

\section{THE BONUS12 RTPC}

The BONuS12 experiment will use a GEM-based RTPC to detect spectator protons. Part of the CLAS12 vertex detectors (SVT and MVT) will be replaced by the $\mathrm{RTPC}$, which is described in the following chapter.

\subsection{TIME PROJECTION CHAMBER}

A Time Projection Chamber (TPC) is a tracking detector consisting of a sensitive volume filled with a gas or a liquid, and with a readout section at the endcaps of the chamber (Fig. 13). When a charged particle is traversing the TPC, the carefully chosen gas (or liquid) will be ionized releasing electrons, called primary electrons. Because of the uniform electric field, created by a cathode and an anode, the electrons will drift towards the positive readout region.

The readout region is composed of the anode (often segmented into pads) and an amplifier to increase the electronic signal - e.g. a wire chamber. All amplifiers work in a similar way. An area with a large electric field accelerates the primary electrons until they acquire enough energy to ionize the gas, hence, creating an electronic avalanche (secondary electrons). The number of electrons is then large enough to create a measurable signal on the readout elements. Generally, the charge deposited by the electrons is measured and recorded, as well as the drift time, that is the time of arrival with respect to an external clock (trigger).

By knowing the drift time, the electric field, the magnetic field, and the pads being hit, charged particle tracks can be reconstructed. If the TPC is inside a magnetic field, the radius of curvature of the track gives access to the ratio $P / q$, and using the timing information, the particle can be identified.

A TPC was used for the first time in the PEP-4 detector at SLAC [9]. Later, it was used in a number of experiments including the DELPHI and ALICE experiments at CERN. 


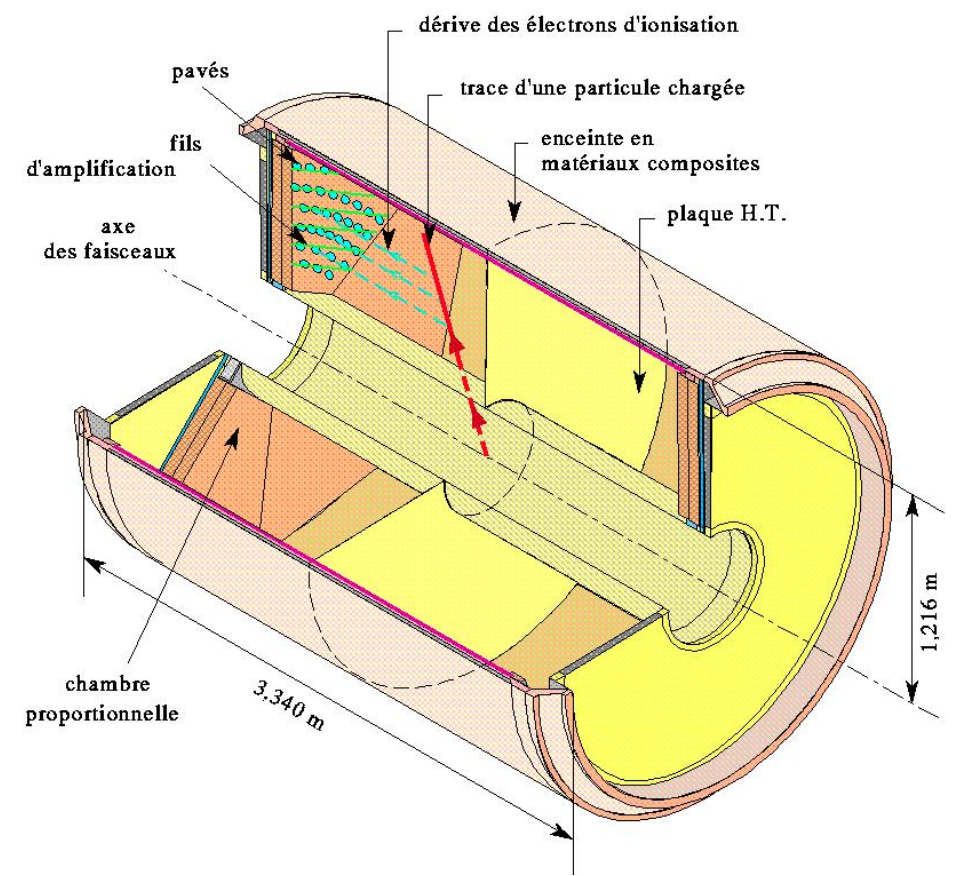

FIG. 13: Cross-section of the DELPHI TPC

\subsection{GAS ELECTRON MULTIPLIER}

Developed at CERN in the late 1990s, Gas Electron Multiplier (GEM) foils are an amplification device that is used in many detectors and have often replaced the Multi-Wire Proportional Chambers (MWPC) in TPCs. A GEM foil is usually composed of a $50 \mu \mathrm{m}$ Kapton foil, coated with $5 \mu \mathrm{m}$ copper layers on both sides. The foil is chemically pierced to reach a high density of holes $\left(50-100 \mathrm{~mm}^{-2}\right)$ [10], see Fig. 14.

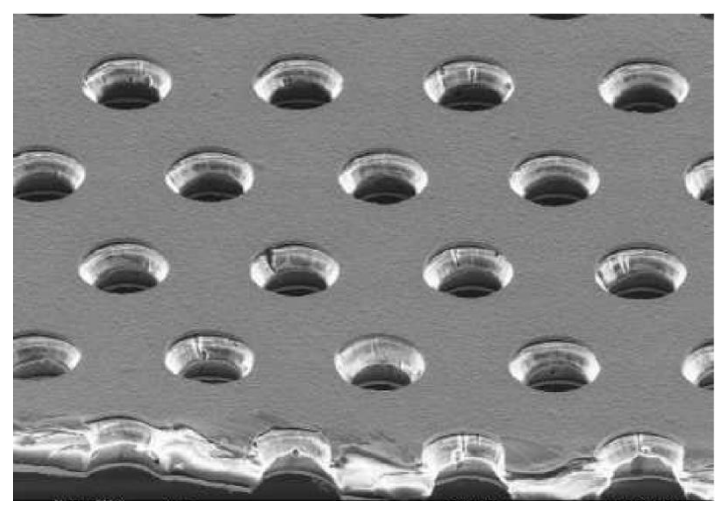

FIG. 14: Picture of a GEM under an electron microscope 
A voltage difference is applied to both sides of the GEM foil creating a large electric field. The field is high enough to create an avalanche of secondary electrons between both sides of the GEM through the holes (see Fig. 15). Usually, the voltage is around $200-250 \mathrm{~V}$, leading to an electron gas gain of around 100 for one foil. Several layers of GEM foils are often stacked with distances of a few millimeters between them to increase the gas gain. The signal is collected on the anode pads without any interference by the newly emitted ions. Indeed, ions, created from ionization in a MWPC, will remain for a long period of time in the gas $(\sim 10-100 \mu \mathrm{s})$ and will interfere with the electric field, reducing its strength and therefore decreasing the gain of the detector. In a GEM, the ions created leave the hole within $\sim 100 \mathrm{~ns}$.

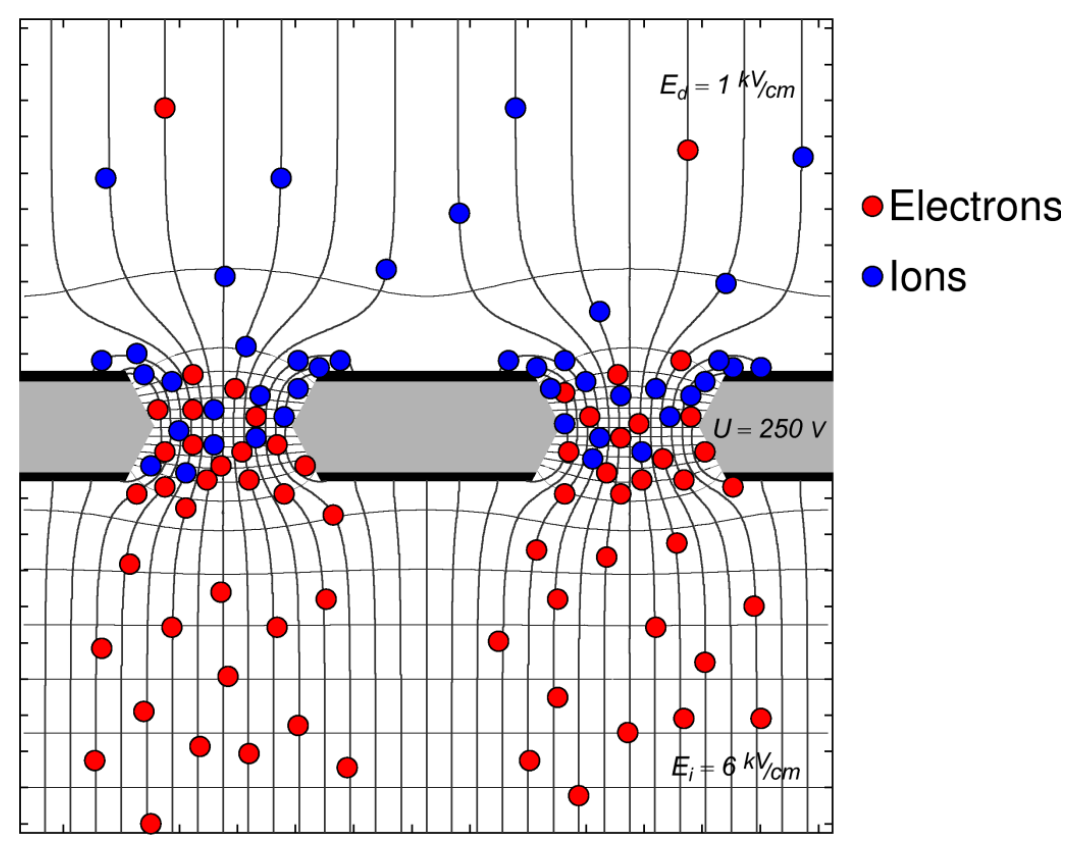

FIG. 15: Electric field near the holes of a GEM

GEM-based detectors are categorized as proportional counters, meaning that the signal read out on the pads is proportional to the incident particle's energy loss. Careful studies of the gas in a specific electric and magnetic field allows for the measurement of the particle's energy loss.

As the gain of a GEM foil is around 100, a stack of three foils is commonly used to reach gains of a few thousand. This configuration is called a triple GEM (see Fig. 16). 


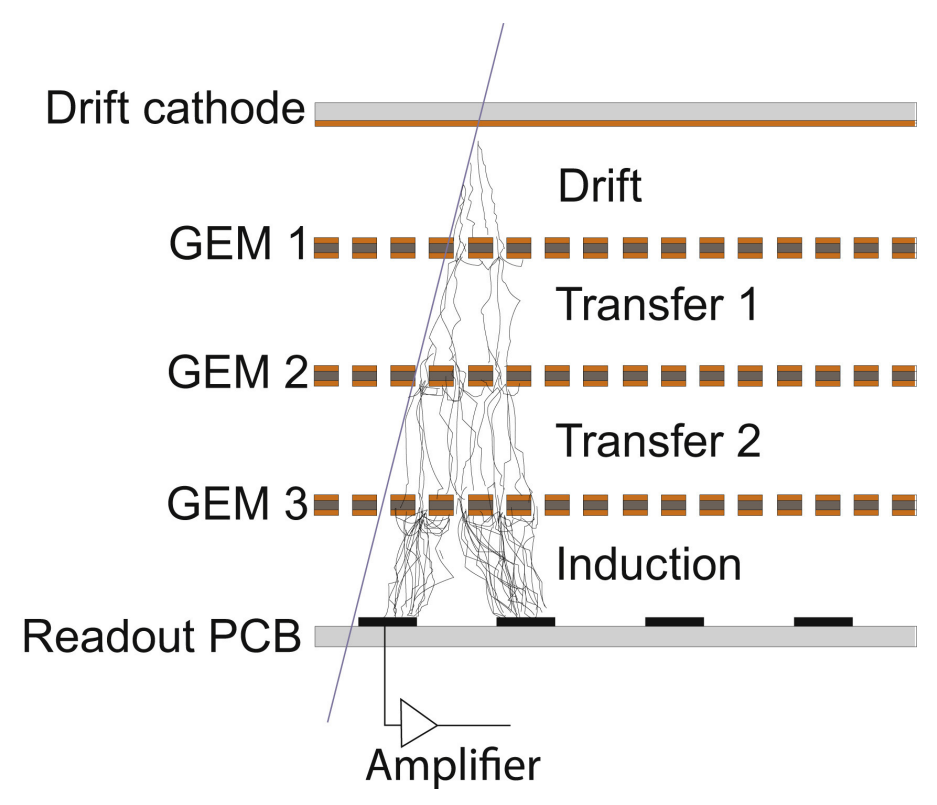

FIG. 16: Triple GEM design

\subsection{RADIAL TIME PROJECTION CHAMBERS}

GEM foils are flexible enough to be bend to form semi-cylinders or cylinders. This feature allows for the readout region to be on the outer cylindrical surface of the TPC, instead of being located at the endcaps. As the electric field is radial in this case, the detector is called a Radial Time Projection Chamber (RTPC).

Such a detector was used by two experiments at Jefferson Lab: BONuS and eg6. Both experiments used very similar designs. Fig. 17 and Fig. 18 show different views of both detectors. The motivation of using a RTPC over a TPC for both experiments are similar. If the readout were located at the endcaps, the drift distance would be about $20 \mathrm{~cm}$, when in the case of the RTPC, it would be around $3 \mathrm{~cm}$. The shorter the distance, the shorter the drift time, meaning that the electronic readout can handle higher rates. Secondly, since more pads can be placed on the cylinder, a higher rate can be achieved without pile-up and potentially a higher resolution.

A new run of the BONuS experiment, the BONuS12 experiment, was proposed and approved using the upgraded CEBAF $11 \mathrm{GeV}$ electron beam and CLAS12 detector. A third RTPC will be built, improving on the two previous RTPCs used in CLAS. 
The triple GEM system of the previous BONuS and eg6 RTPCs is composed of six GEM foils bent in two hemispheres and glued at the poles (see Fig. 17), creating two dead areas at the poles.

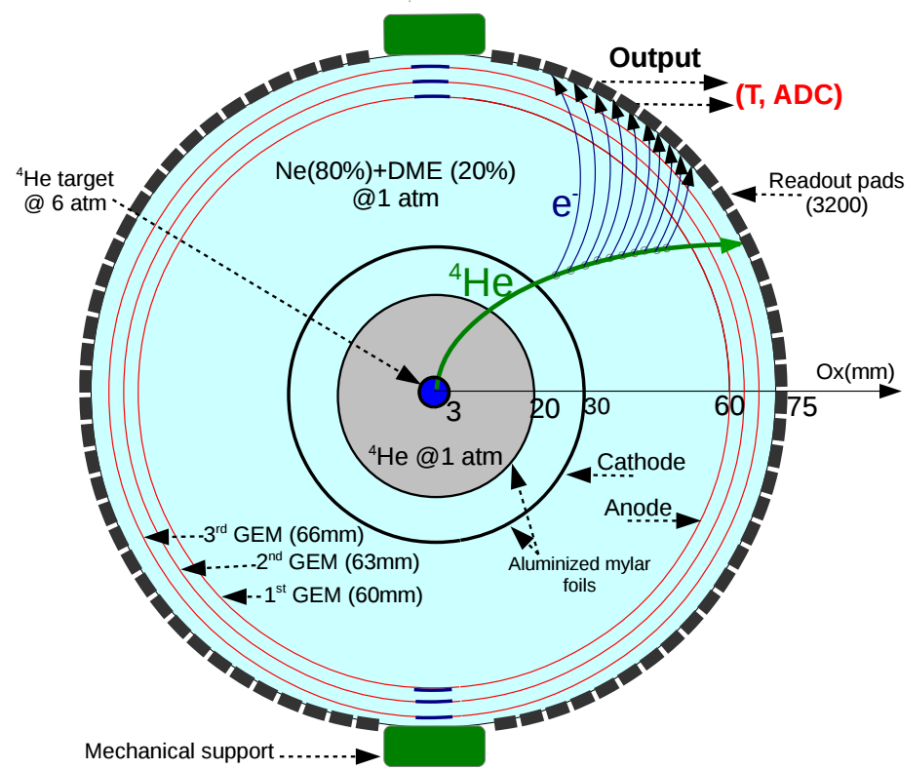

FIG. 17: Cross section of the eg6 RTPC (along the beamline)

From the interaction point to its outer part, the different regions of the RTPCs are detailed as followed [11]:

- the target is made of a $3 \mathrm{~mm}$ radius Kapton straw, filled with deuterium at 7 atm for BONuS, and helium-4 at 6 atm for eg6

- a buffer region is located from $3 \mathrm{~mm}$ to $20 \mathrm{~mm}$ and filled with helium-4 at 1 atm. This gap ensure that Møller electrons from the beam are escaping in the forward direction and do not reach the sensitive volume of the RTPC. Those electrons are deflected because of the solenoid magnet around the detector

- a second buffer region is located from $20 \mathrm{~mm}$ (ground aluminized Mylar window) to $30 \mathrm{~mm}$ (aluminized Mylar cathode) and is filled with a gas mixture of $80 \%$ helium / 20\% DME (dimethyl ether) for BONuS and 80\% Ne / 20\% DME 
- the drift region, filled with the same gas, is located from $30 \mathrm{~mm}$ to $60 \mathrm{~mm}$. This is where the spectator proton ionizes the gas and drifts towards the readout region due to the electric field $(\sim 500-550 \mathrm{~V} / \mathrm{cm})$

- the amplification region is composed of 3 layers of GEMs, located at 60, 63 and $66 \mathrm{~mm}$. The electron signal is amplified by a factor $10^{3}$

- the readout pads are etched onto a printed-circuit board with metal traces which is located at $69 \mathrm{~mm}$. The pads, measuring 5 by $4.5 \mathrm{~mm}$ each, are arranged by rows which are shifted with respect to each other to identify tracks (track angles) with better precision.

A schematic of the BONuS RTPC and a reconstructed track in the BONuS RTPC are shown in Fig. 18 and Fig. 19.

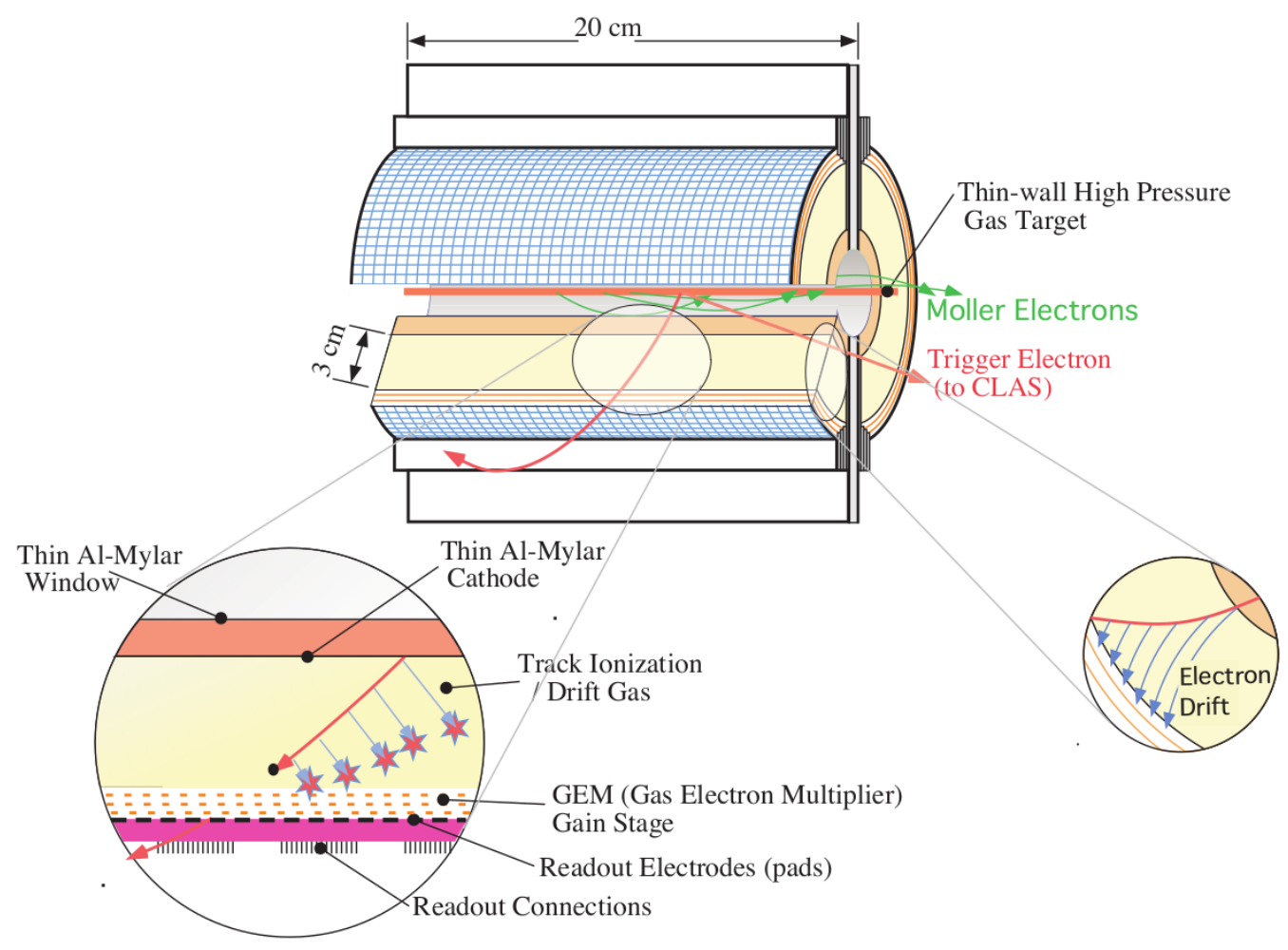

FIG. 18: BONuS RTPC 


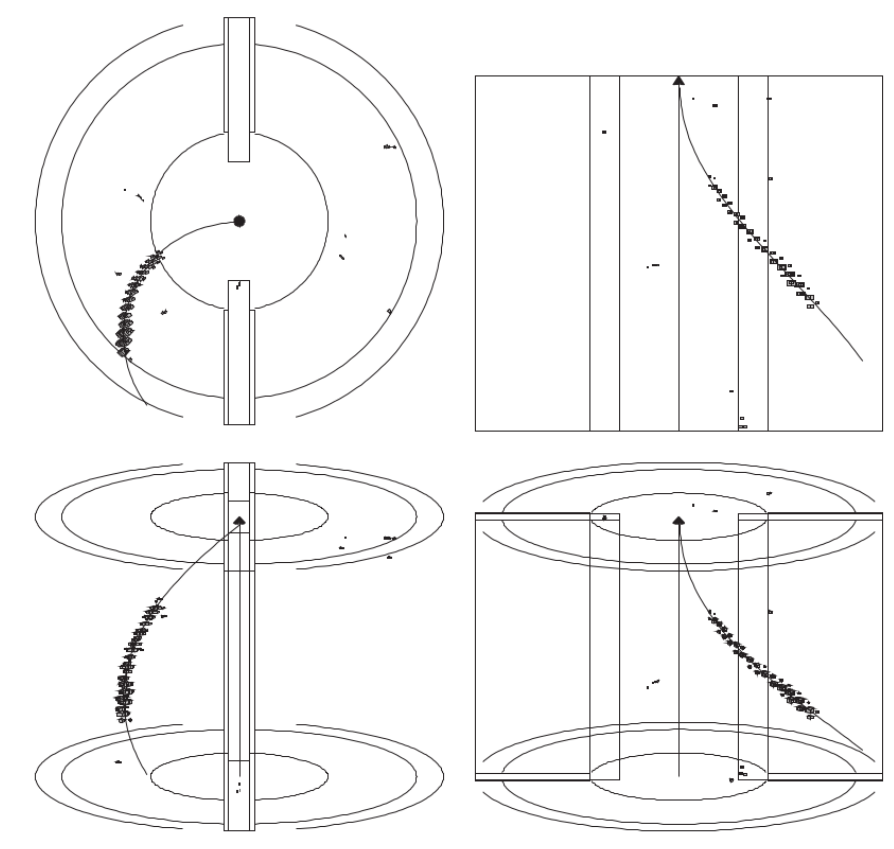

FIG. 19: Reconstructed track of a spectator proton with the BONuS RTPC

\subsection{PREVIOUS READOUT ELECTRONICS}

The BONuS and eg6 experiments used the same electronics to read and digitize the analog signals of the RTPCs. The Front End Card (FEC) is composed of preamplifier/shaping amplifier chips (PASA) and the CERN ALTRO cards developed and used for the ALICE experiment TPC. Each bundle of PASA and ALTRO can handle 16 channels and 8 of them form a FEC (128 channels per card).

In order to keep the data size manageable, the ALTRO card has a zero-suppression mode, allowing to set a certain threshold, just above the noise level. Three samples (of $114 \mathrm{~ns}$ ) before and after a signal exceeding that threshold were saved to fit the shape of the signal better.

The BONuS RTPC used the ALTRO readout controller (U2F) to control and transfer the data from the FECs. It also imports the trigger and clock from the CLAS acquisition system and can transfer the data via USB 2.0 or VME interface. For this readout configuration, the Data Acquisition System (DAQ) rate was about $500 \mathrm{~Hz}[11]$ (see Fig. 20). 


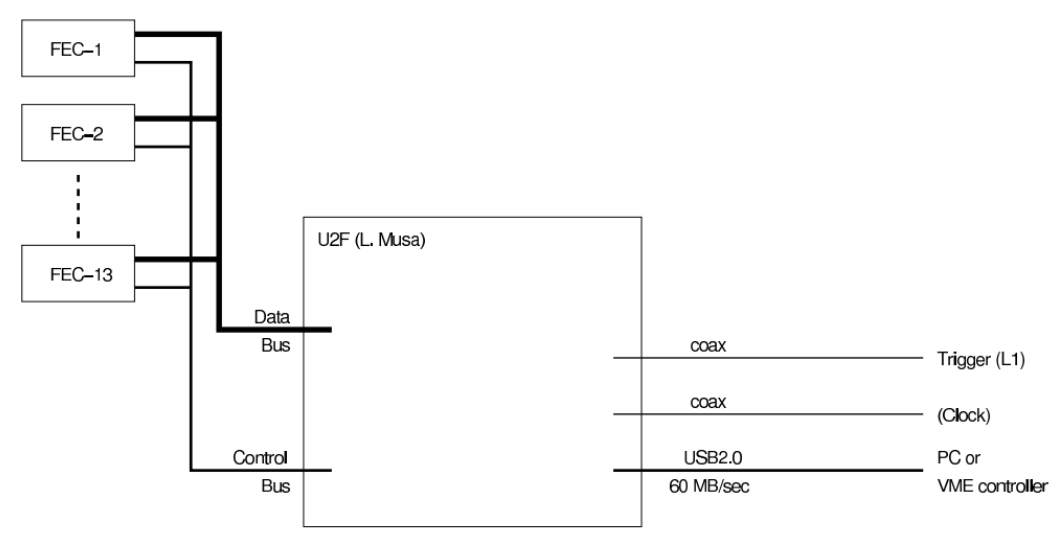

FIG. 20: Diagram of the BONuS readout

The eg6 RTPC improved the previous readout by replacing the U2F controller with Readout Control Units (RCU) being connected optically to the CLAS DAQ system $(200 \mathrm{MB} / \mathrm{s}$ link). With the new RCU, the experiment operated with a rate of $3.1 \mathrm{kHz}$ for a luminosity around $\mathcal{L}=10^{34} \mathrm{~cm}^{-2} \mathrm{~s}^{-1}$ (see Fig. 21).

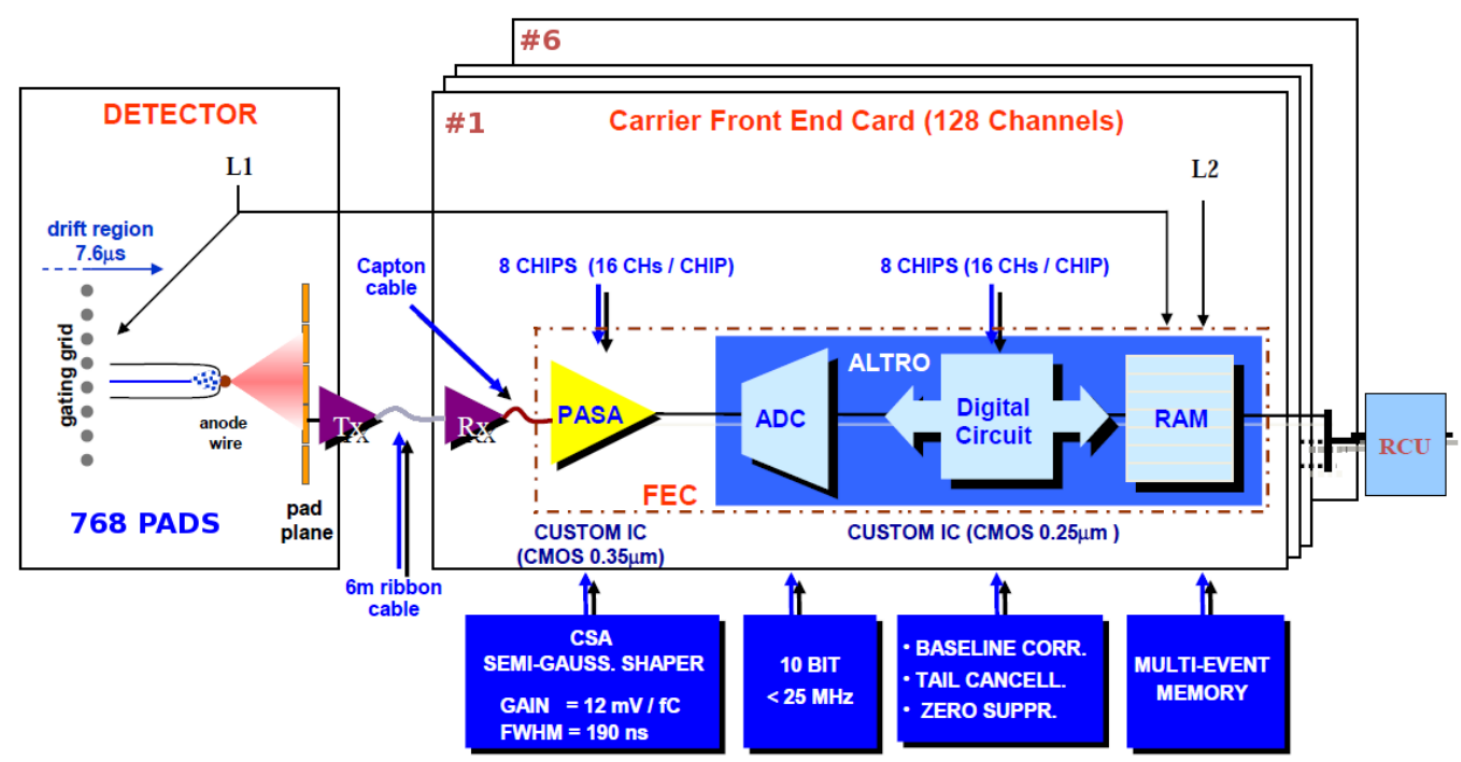

FIG. 21: Structure of the FEC core logic used by eg6 


\subsection{BONUS12 RTPC}

The design of the BONuS12 RTPC will be an improvement of the previous two RTPCs with the following elements being changed:

- length: the BONuS12 RTPC will be $40 \mathrm{~cm}$, twice as along as the one used in the $6 \mathrm{GeV}$ area

- pad size: the pads will be smaller, 4 by $2.7 \mathrm{~mm}$, improving the resolution of the detector

- the first layer of GEM will be located at $70 \mathrm{~mm}$, increasing the drift region to $4 \mathrm{~cm}$

- the dead area due to the glueing of the GEM foils will be reduced only to 3 $\mathrm{mm}$

- a new readout electronics, which will sustain higher rates.

Using the GARFIELD++ [12] and MAGBOLTZ [13] software, several gas mixtures have been studied ${ }^{1}$. Two plots summarize the results. The drift time $\left(t_{d}\right)$ as a function of the angle traveled $\left(\Delta \phi_{d}\right)$ by electrons being ionized $20 \mathrm{~mm}$ away from the first GEM foil were calculated for different gases and mixture (see Fig. 22). A shorter drift time is desirable to allow a higher rate. A small difference in $\phi$ also allows to accept a higher rate, as less pads will be hit. Similarly, the variability $\sigma$ due to diffusion of both quantities were plotted (see Fig. 23).

From these plots, it appears that $\mathrm{He} / \mathrm{CO}_{2}$ mixtures are a good choice. The exact concentration $\mathrm{He} / \mathrm{CO}_{2}(70 \% / 30 \%$ or $80 \% / 20 \%)$ will be decided when the studies of the energy loss will be completed.

\footnotetext{
${ }^{1}$ Studies by Nathan Dzbenski
} 


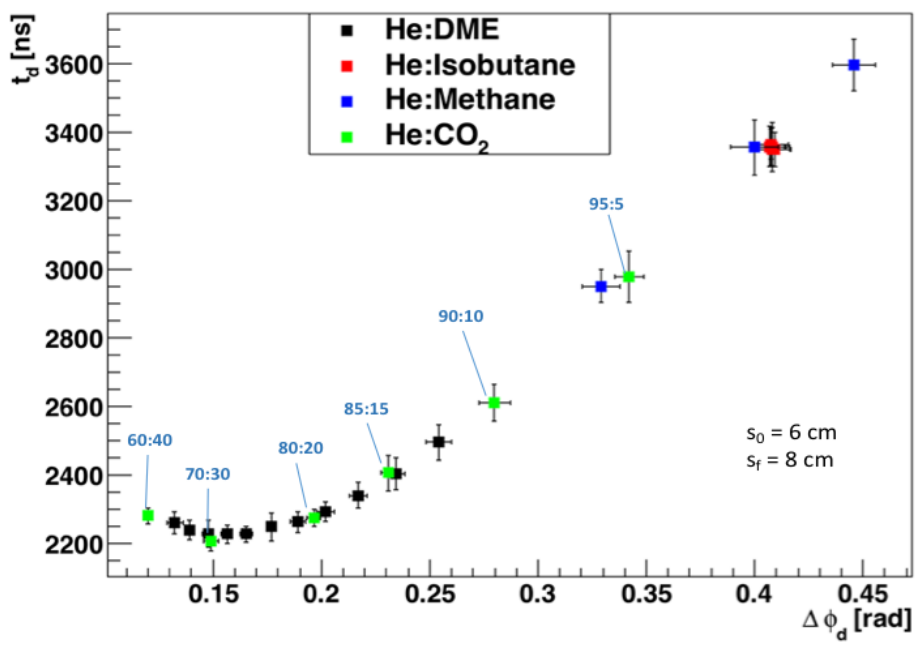

FIG. 22: $t_{d}$ vs. $\Delta \phi_{d}$ for different gas mixtures

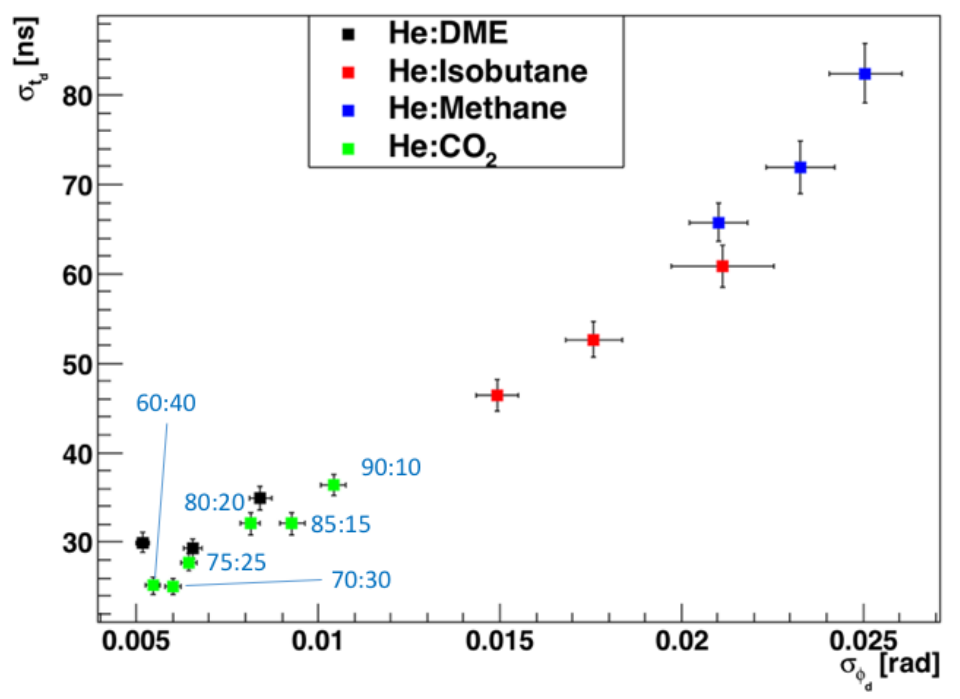

FIG. 23: $\sigma\left(t_{d}\right)$ vs. $\sigma\left(\phi_{d}\right)$ for different gas mixtures 


\section{CHAPTER 4}

\section{NEW READOUT ELECTRONICS}

\subsection{THE DREAM CHIP}

The BONuS12 experiment plans to use the existing DREAM ${ }^{1}$ electronics cards developed by CEA-Saclay. The electronics was primarily developed for the Micromegas detector of CLAS12. The DREAM chip consists of three main elements, a Charge Sensitive preAmplifier (CSA), an analog filter and a Switched Capacitor Array (SCA) which is a 512 cell circular buffer [14] (see Fig. 24). Each component is tunable to match the readout conditions of experiments (see Table 3). Finally, the analog data from DREAM is exported to an Analog-to-Digital Converter and a Field-Programmable Gate Array (FPGA). The DREAM chips are placed on Front End Units (FEU).

Each FEU hosts 8 DREAM chips capable of handling 64 channels. The triggered signals are read-out asynchronously and continuously allowing a "deadtime free" operation. The FEU are optically connected to a Back End Unit (BEU), part of the JLab DAQ system, which provides the trigger, slow control and exports the data for storage (see Fig. 25).

The digital part of the FEU is able to realize a number of data manipulations before sending out the data: pedestal equalization, common mode noise subtraction, and zero suppression. [15] (see Fig. 26)

\footnotetext{
${ }^{1}$ Dead-timeless Read-out Electronics ASIC for Micromegas (ASIC: Application-Specific Integrated Circuit)
} 


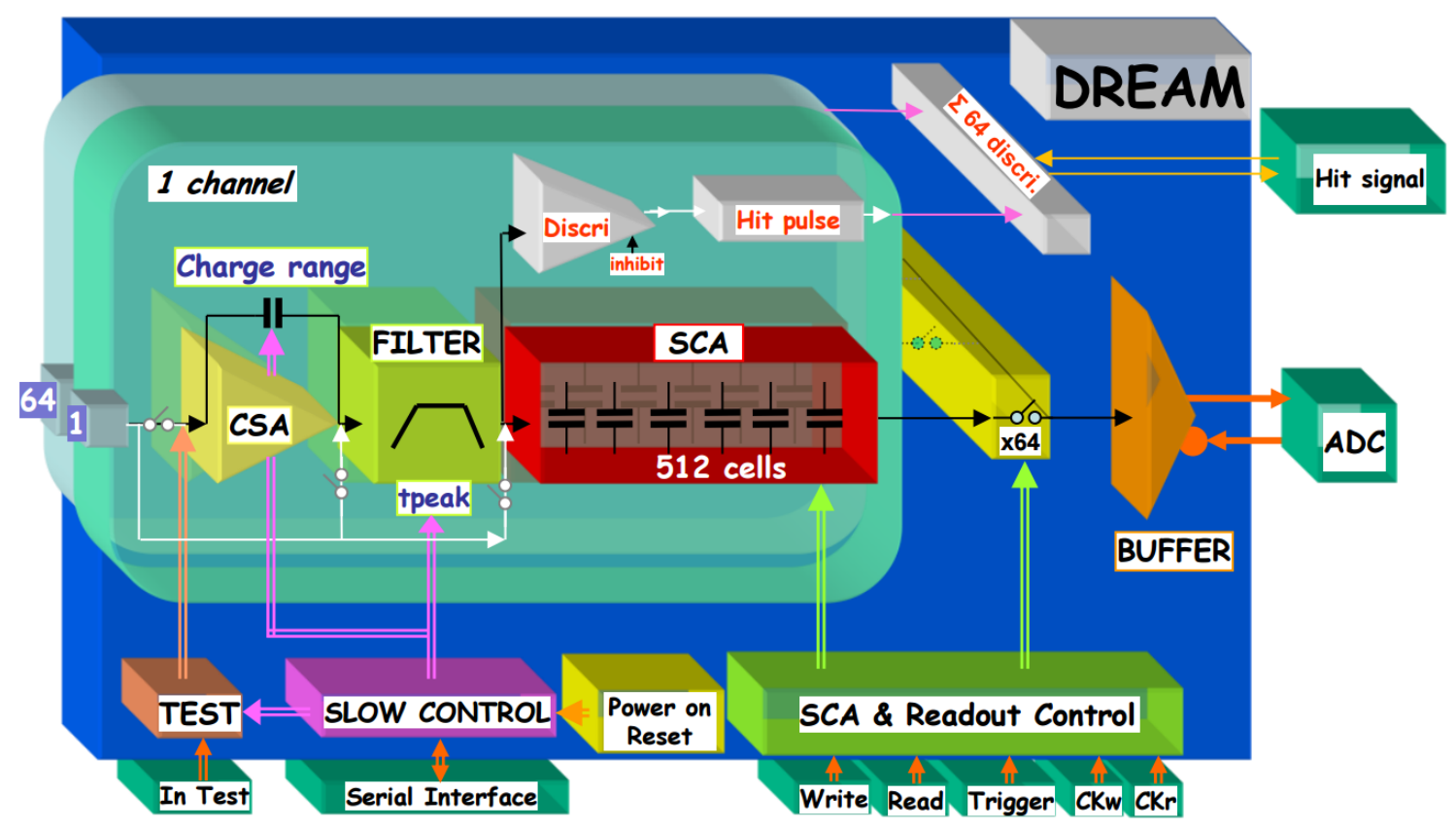

FIG. 24: Block diagram of the DREAM chip [14]

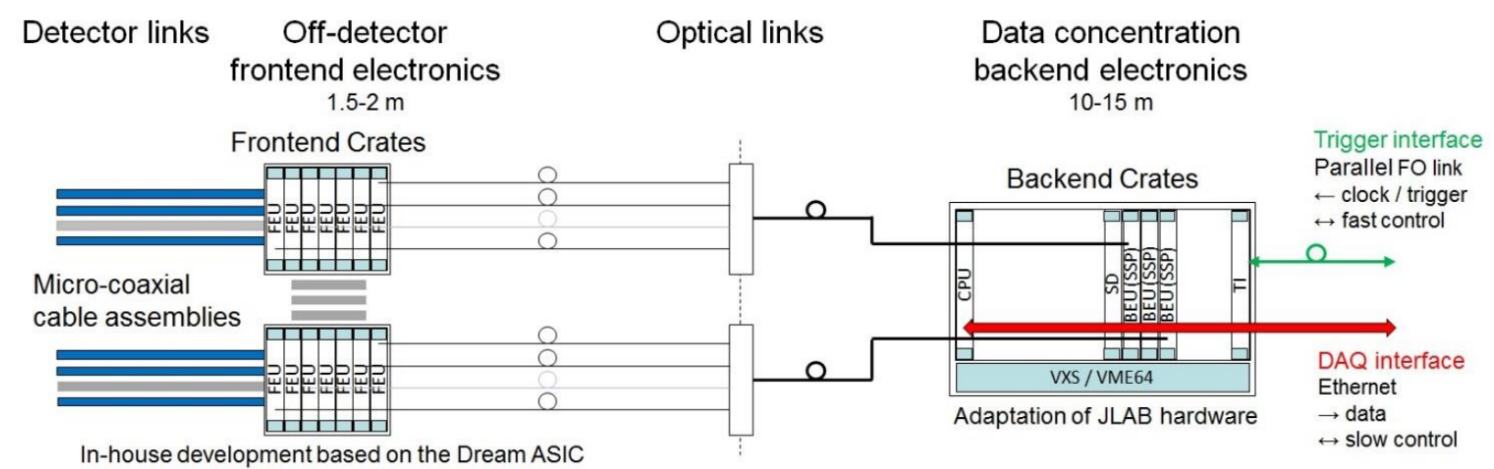

FIG. 25: DREAM electronics readout architecture [15] 
TABLE 3: Summary of the DREAM requirements [14]

\begin{tabular}{ll}
\hline \hline Parameter & Value \\
\hline Polarity of detector signal & Negative or Positive \\
Number of channels & 64 \\
External Preamplifier option & Yes; access to the filter or SCA inputs \\
\hline Charge measurement & \\
$\quad \begin{array}{l}\text { Input dynamic range/gain } \\
\text { Output dynamic range }\end{array}$ & $50 \mathrm{fC} ; 100 \mathrm{fC} ; 200 \mathrm{fC} ; 600 \mathrm{fC}$, selectable by channel \\
Integral NonLinearity (INL) & $<2 \%$ \\
Charge Resolution & $>8$ bits \\
\hline Sampling & $50 \mathrm{~ns}$ to $900 \mathrm{~ns}(16$ values $)$ \\
Peaking time value & 512 \\
Number of SCA Time bins \\
Sampling Frequency (WCk) & $1 \mathrm{MHz}$ to $50 \mathrm{MHz}$ \\
\hline Triggering & \\
Discriminator solution & Leading edge \\
HIT signal & OR of the 64 discriminator outputs in LVDS level \\
Threshold Range & $5 \%$ or $17.5 \%$ of the input dynamic range \\
INL & $<5 \%$ \\
Threshold value & $(7$-bit + polarity bit) Digital-to-Analog Converter \\
Minimum threshold value & common to all channels \\
$\geq$ noise \\
\hline Readout & Up to $20 \mathrm{MHz}$ \\
Readout frequency & all channels excepted those disabled (statically) \\
Channel Readout mode & Triggered columns only \\
SCA cell Readout mode & Up to $20 \mathrm{kHz}(4$ samples read/trigger) \\
\hline Trigger rate &
\end{tabular}




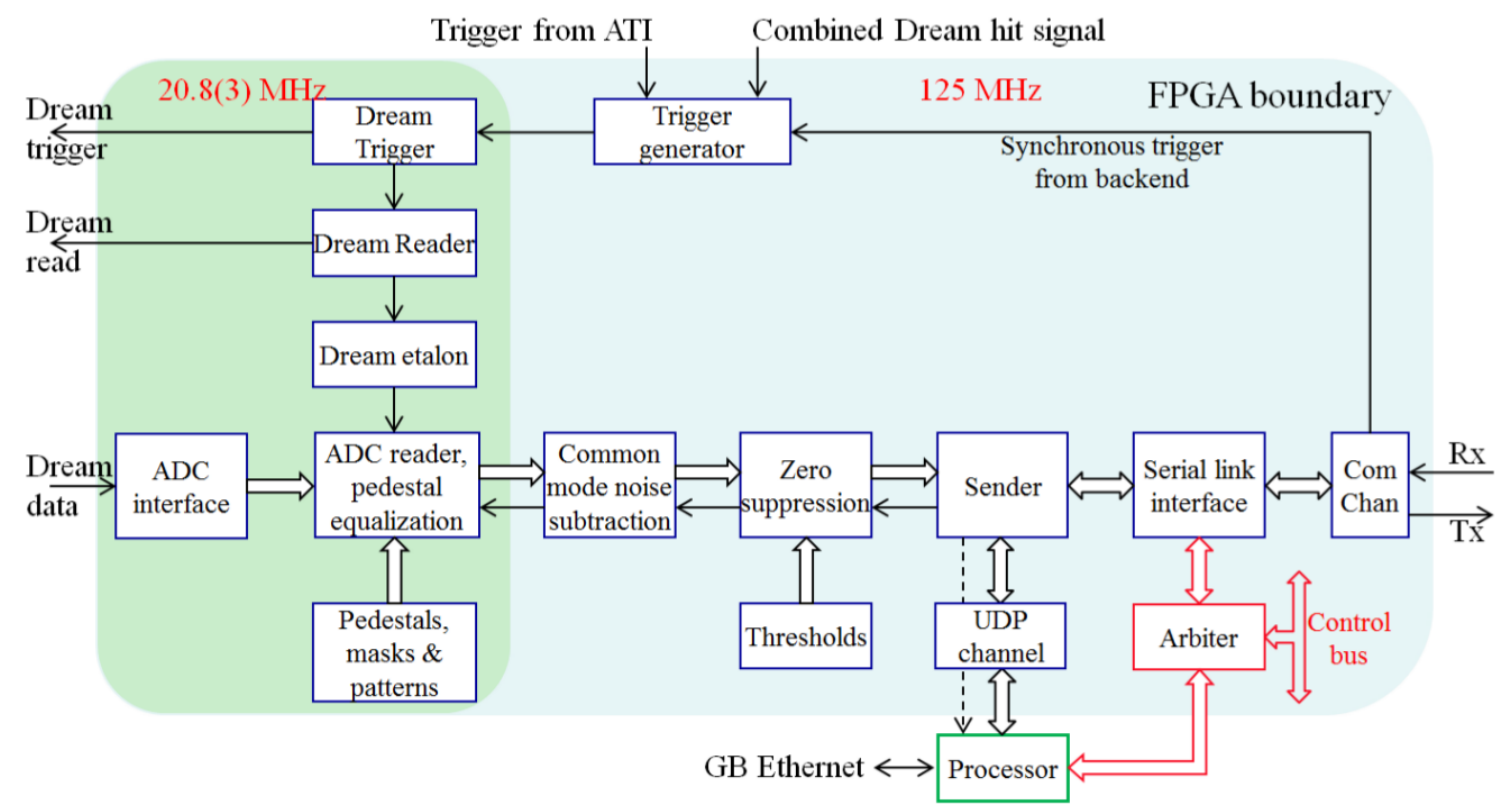

FIG. 26: Modular structure of the FEU core logic [15]

\subsection{TEST BENCH}

A working prototype of the eg6 RTPC was brought to ODU in order to test the DREAM electronics, and to check if it can be used with a RTPC that matches BONuS12 needs. The ODU test bench has the capabilities to mix two different gases and supply the mixture to the RTPC. However, for the following tests, a premixed gas of $90 \% \mathrm{He} / 10 \% \mathrm{CO}_{2}$ was used. Fig. 27 shows a front and back pictures of the setup.

The test bench is composed of the following elements:

- the RTPC

- a signal cable adaptation board

- a signal cable

- a crate with FEU boards

- HV and LV power supplies

- radioactive source 

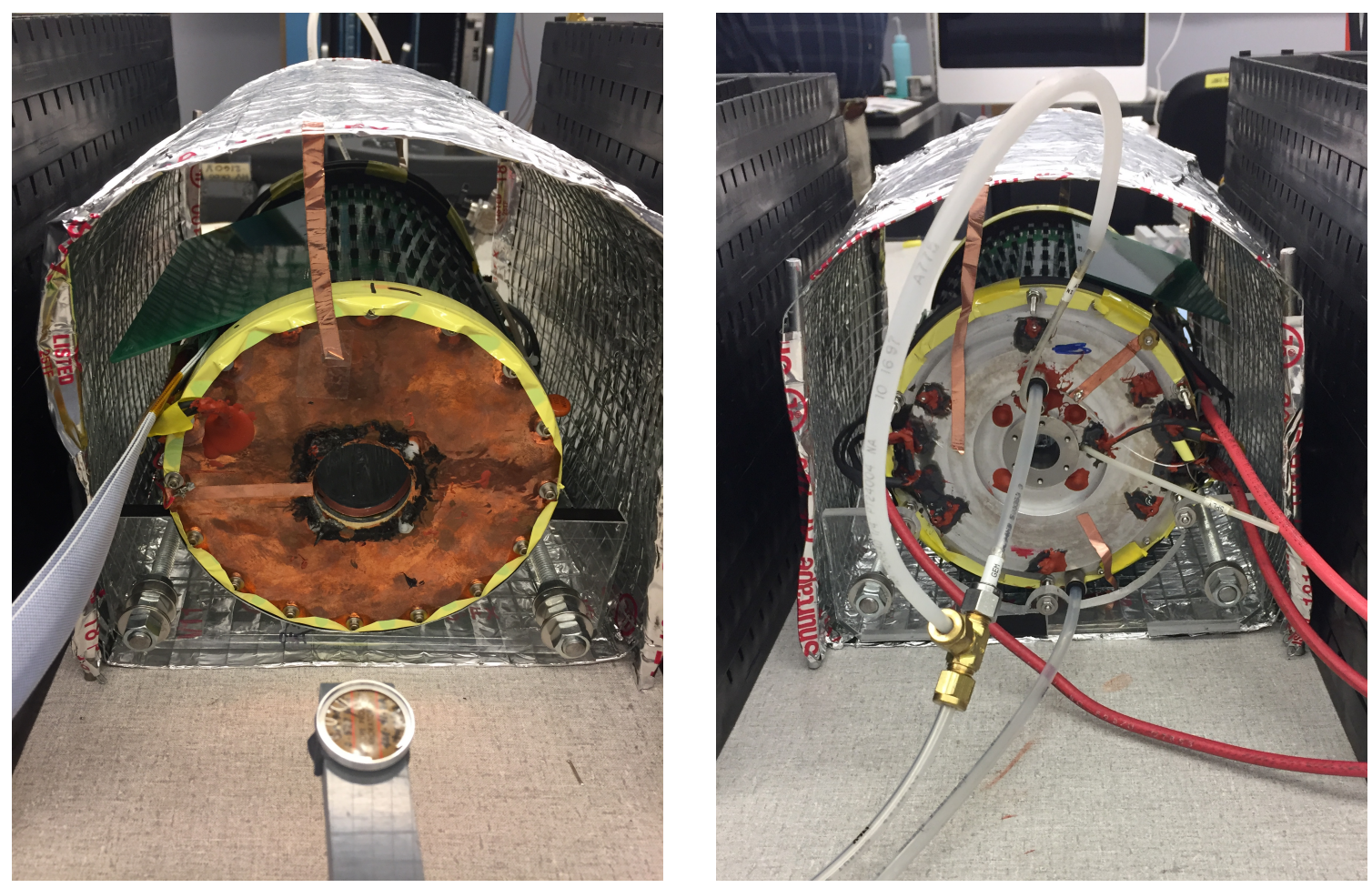

FIG. 27: Front and back pictures of the ODU test bench

The high voltages were provided by two power supplies: one for the cathode and another one for each hemisphere of GEM foils. A voltage divider ensures that each GEM is at the right potential.

A crate hosting five FEUs was received and installed. Since no BEU is available, the crate controls the FEUs and export the data via USB to a local computer. On the computer is installed the Xilinx software suite necessary to control the FPGA inside the FEU, as well as a custom software suite from CEA to control DREAM (see Fig. 28). The crate and the FEUs are powered by a low voltage power supply set to $5 \mathrm{~V}$ and delivering a current of $2.2 \mathrm{~A}$.

The connection between the RTPC and the FEU required an adaptation card. A printed-circuit board was designed with the KiCAD software, and connectors were soldered on it. The card links four M50-391042 connectors, which match the output from RPTC $(2 \times 8$ pads per connector), to a MEC8-135-02-L-D-RA1 connector, corresponding to the micro-coaxial cable connected to the input FEU (Figure 29). Since 


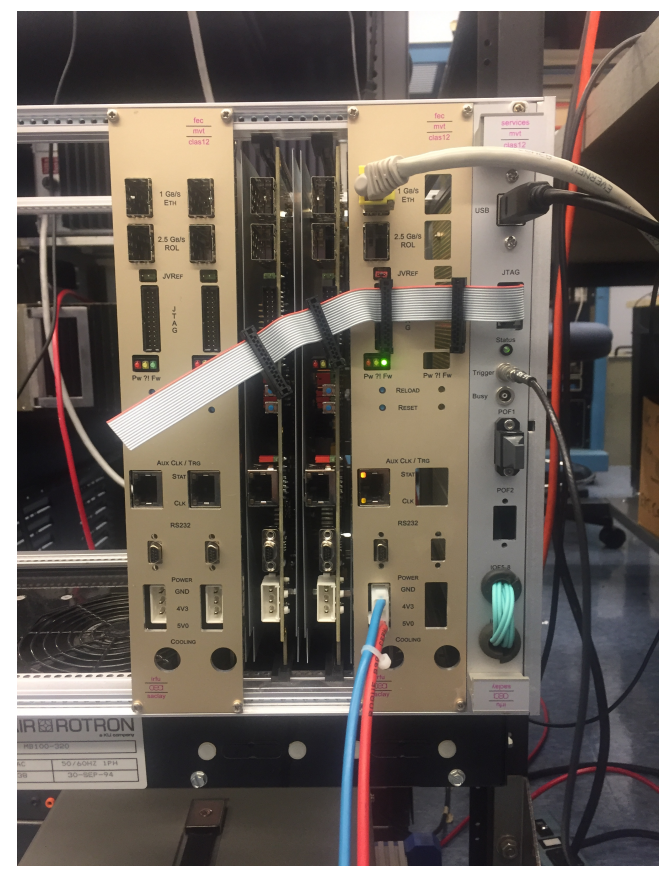

FIG. 28: Create hosting the FEUs

the FEU used is protected against over-current (due to discharges inside the RTPC, for example), the card did not need a protection circuit. For the actual experiment, the FEU will not be protected and protected cards will need to be designed.

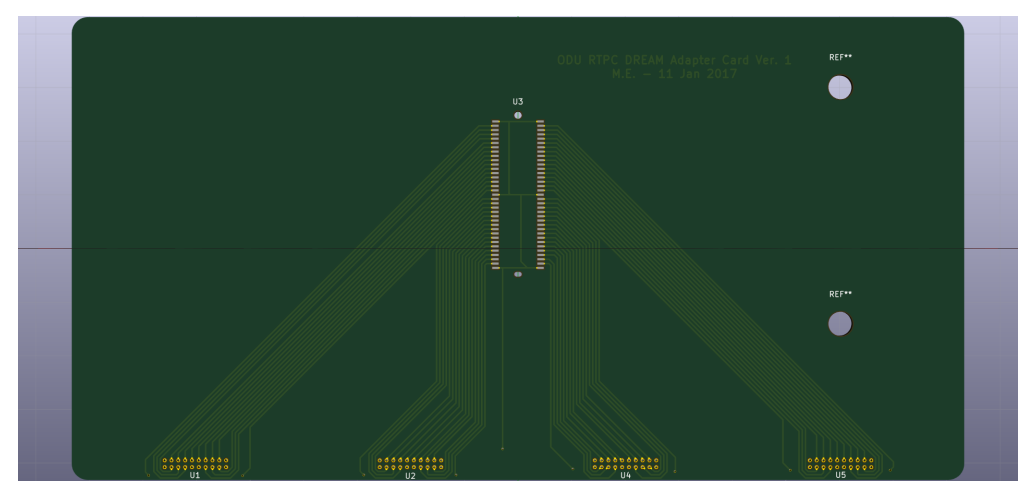

FIG. 29: 3D drawing of the ODU RTPC adaptation card 
In order to characterize the detector and configure the electronics correctly, several tests were made. First, the pedestal values for the 64 channels were measured, then the offset of the chip was determined and finally, signals were acquired from a radioactive source. The results are presented in the next sections of this chapter.

\subsection{DREAM CONFIGURATION}

In its current configuration, the DREAM chip can take consecutive 48 ns samples. The time between the trigger and the data read-out was unchanged and fixed to center the signal on the time window.

The DREAM acquisition software requires a configuration file which allows the user to tune the FEU and DREAM to the experimental constraints. The relevant parameters for our tests are:

- Feu * Main_Conf_Samples is the number of samples to be read per trigger (e.g. the time window for the tests is $2.4 \mu \mathrm{s}-50$ samples $\cdot 48 \mathrm{~ns}=2.4 \mu \mathrm{s}$ )

- Feu * Feu_PreScale_EvtData limits the output of data send by the FEU to the BEU. Only event every $n^{\text {th }}$ events will be transferred and saved

- Feu * Trig_Conf_Src sets the source of the trigger. For the tests only two parameters were relevant:

- Tg_Src_NegExp internally generates a random trigger

- Tg_Src_PushButton is the self triggering option. A trigger will be generated for every hit above a set threshold

- Feu * Trig_Conf_Rate sets the trigger rate generation when in Tg_Src_NegExp: $0-20 \mathrm{~Hz}, 1-200 \mathrm{~Hz}, 2-2 \mathrm{kHz}, 3-20 \mathrm{kHz}$

- Feu * Trig_Conf_TrigPipeLen sets the delay between the two trigger signals produced by the trigger generator. $n$ between 1 and 4095, delay will be equal to $n \cdot 8 \mathrm{~ns}$

- Feu * Main_Conf_SparseRd is the sparse readout factor:

- 0 read all samples

- $n=[1,7]$ skip $n$ sample 


\subsection{NOISE STUDIES}

Before starting data taking with a radioactive source, the pedestals of each channel were measured. 200 events were taken with five different settings to evaluate the noise from each source: 1) cable only, 2) cable and adaptation card, 3) cable and adaptation card plugged on the RTPC (no high voltage), 4) cable and adaptation card plugged on the RTPC (cathode HV: -4000 V, HV GEMs: -2500 V), and 5) cable and adaptation card plugged on the RTPC (cathode HV: -4350 V, HV GEMs: $-2850 \mathrm{~V})$.

Fig. 30 shows the $\sigma$ (noise) for the channels where the card was connected to. Some of the pins of the connectors were not soldered properly, hence some channels show very low noise or no noise at all, when connected to the RTPC.

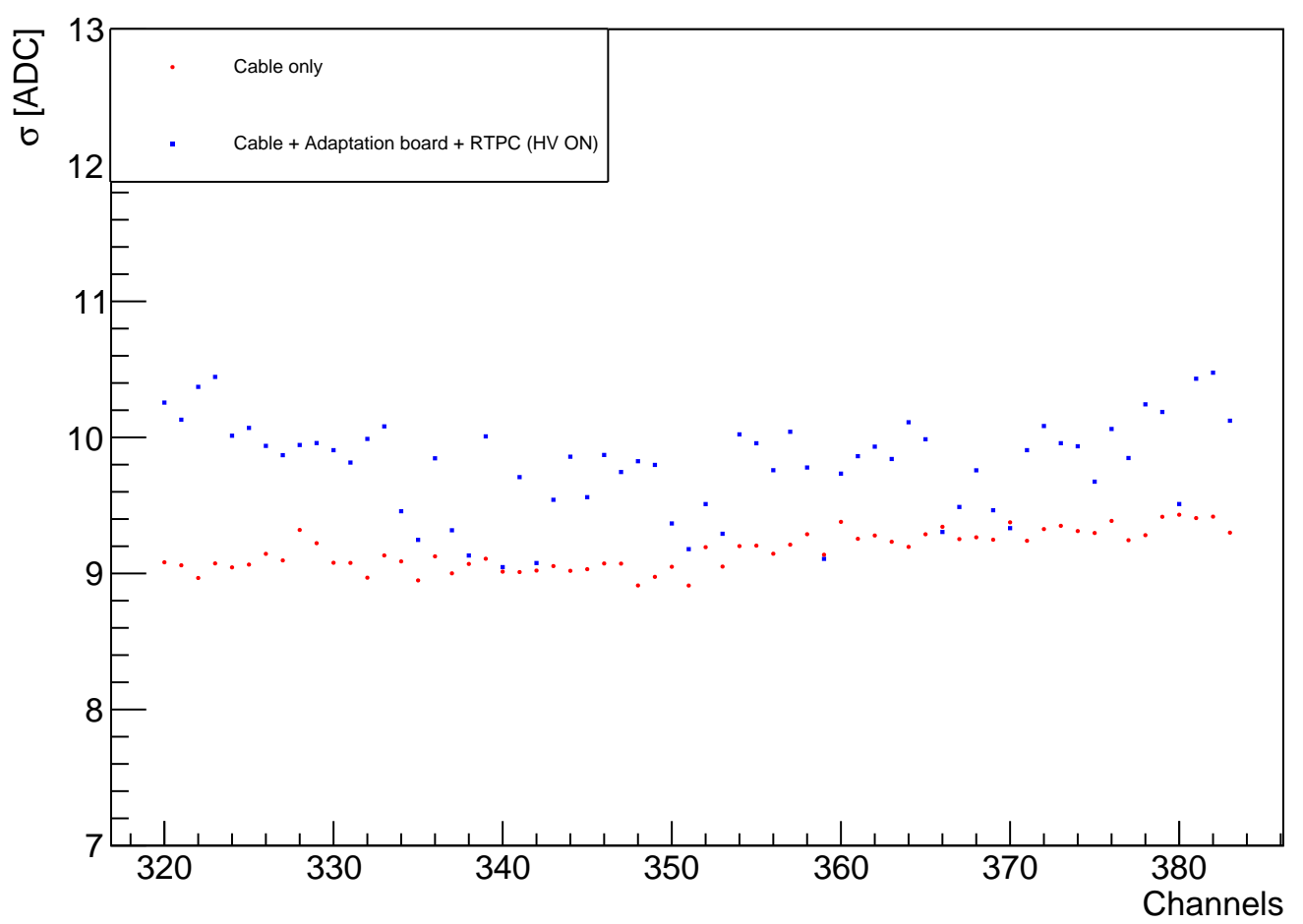

FIG. 30: Noise levels for the channels connected to the RTPC

We can conclude that very little noise was added by the RTPC as the full scale of the ADC is 4096 and the noise due to all elements is below 11 for all channels. 


\subsection{DATA WITH A SOURCE}

A strontium-90 source (Sr-90) was used to take data, which will be analyzed to get the shape of a RTPC signal with the DREAM electronics. Sr-90 decay into yttrium-90 and an electron, with a decay energy of $0.546 \mathrm{MeV}$. The signals have to be fitted and the fit will be used in the simulations. There are also secondary decay electrons up to about $2.2 \mathrm{MeV}$.

The FEU was configured so that any signals above a threshold (carefully chosen) will trigger one event and will be recorded. It is also capable of performing pedestal equalization while taking data. A pedestal file needs to be generated and can be added as an argument when running the acquisition software. However for this run, no pre-generated pedestal file was used.

Two hundred events were recorded and underwent a series of various corrections before being fitted. First, the pedestals for each channel were calculated by taking the average of ADC from sample 35 to 50, and were subtracted. To avoid negative signals, a constant, 50 in the present case, was added to the pedestals. Secondly, using the $\sigma$ calculated earlier, only signals above $3 \sigma$ were kept. Fig. 31 and Fig. 32 are two examples of good signals.

The first result is that DREAM works with RTPCs. Knowing the length of the signal is necessary to determine the maximum acquisition rate. The shape of the signal can be fitted and will be used in the simulation. 


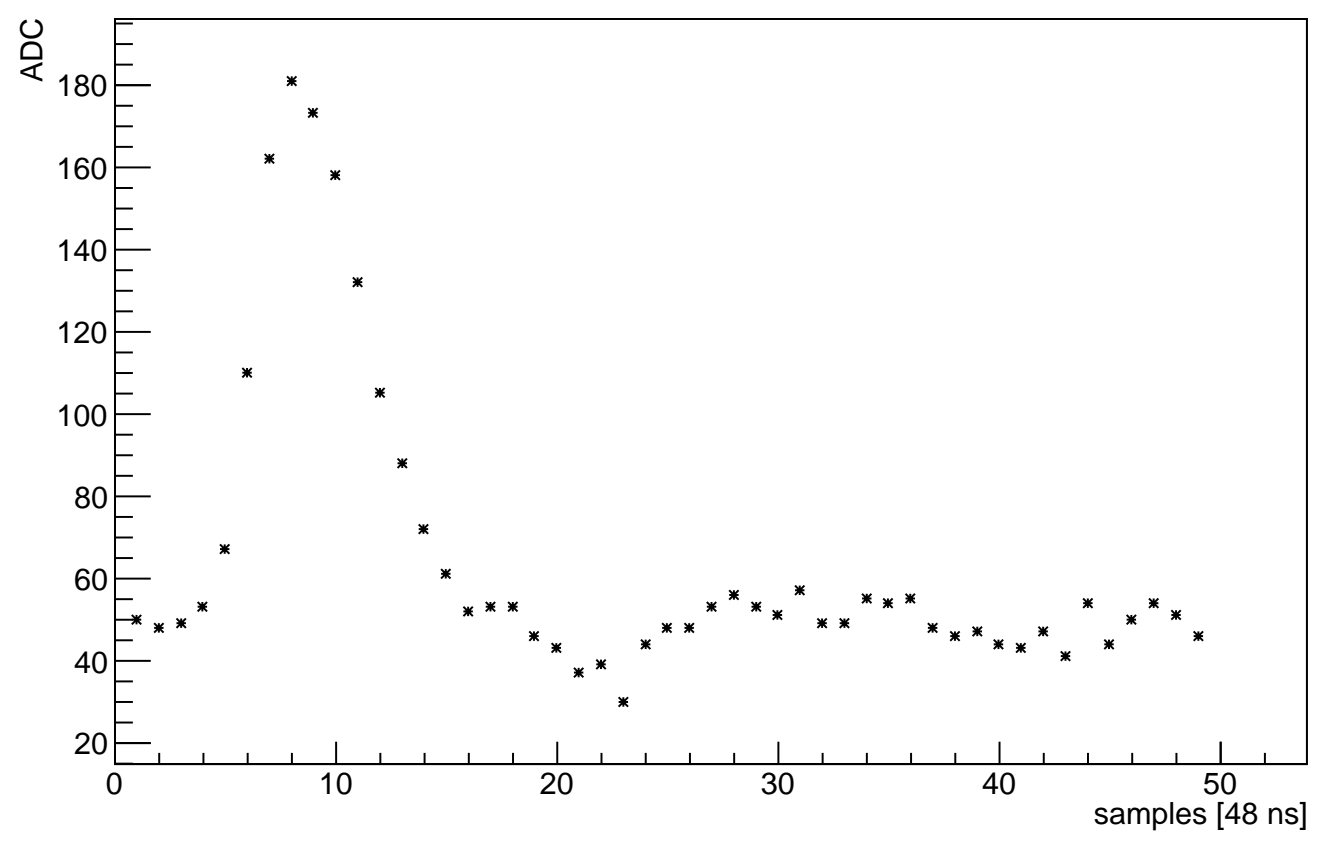

FIG. 31: Signal from channel 9 (event 17)

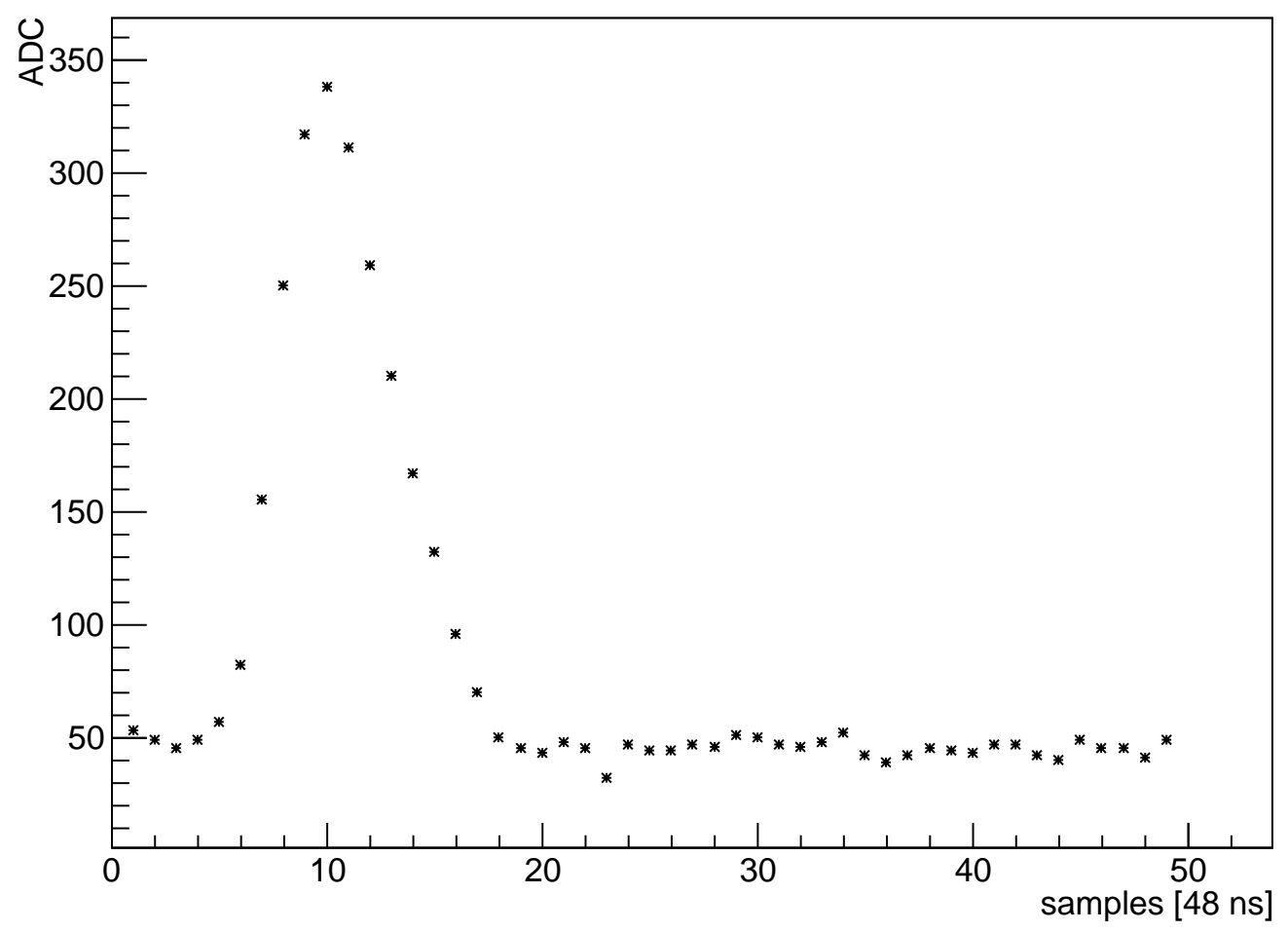

FIG. 32: Signal from channel 35 (event 99) 


\subsection{SIGNAL FIT}

There is no evident way to fit the RTPC signal, therefore various fitting functions have to be investigated. Two different functions were used to fit the signals, acknowledging that more functions could fit the shape of the signal. The ROOT fitting parameters scheme is used to define the functions $(\mathrm{p} 0, \mathrm{p} 1, \ldots)[16]$ :

- "Double Gaussian"

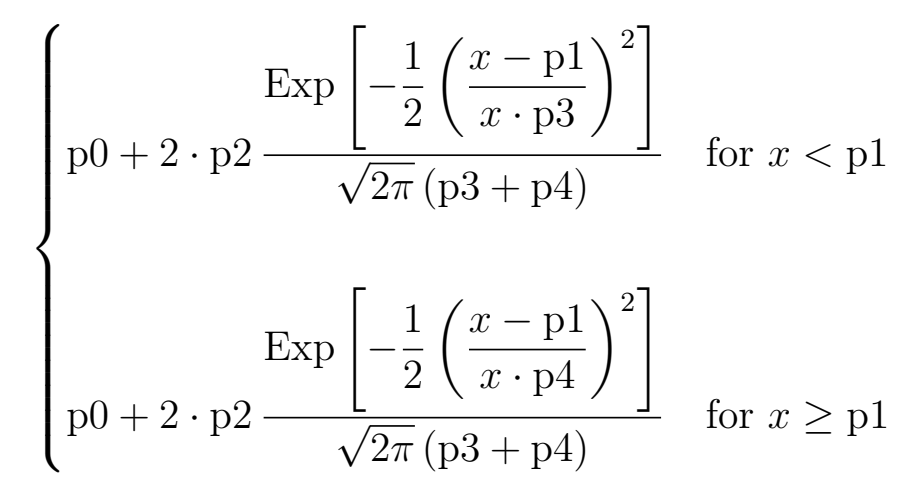

- Skew normal distribution

$$
c+\frac{\mathrm{p} 0}{\mathrm{p} 1} \frac{1}{\sqrt{2 \pi}} \operatorname{Exp}\left[-\frac{\left(\frac{x-\mathrm{p} 2}{\mathrm{p} 1}\right)^{2}}{2}\right]\left[1+\operatorname{Erf}\left(\frac{\mathrm{p} 3 \frac{x-\mathrm{p} 2}{\mathrm{p} 1}}{\sqrt{2}}\right)\right]
$$

where $c$ is a constant set during pedestal substraction (here $c=50$ )

The accuracy of the fit can be checked by looking at the ratio $\chi^{2} /$ ndf. Low values of this ratio indicate a good fit. However the missing error bars on individual data points limits the interpretation of $\chi^{2}$. 


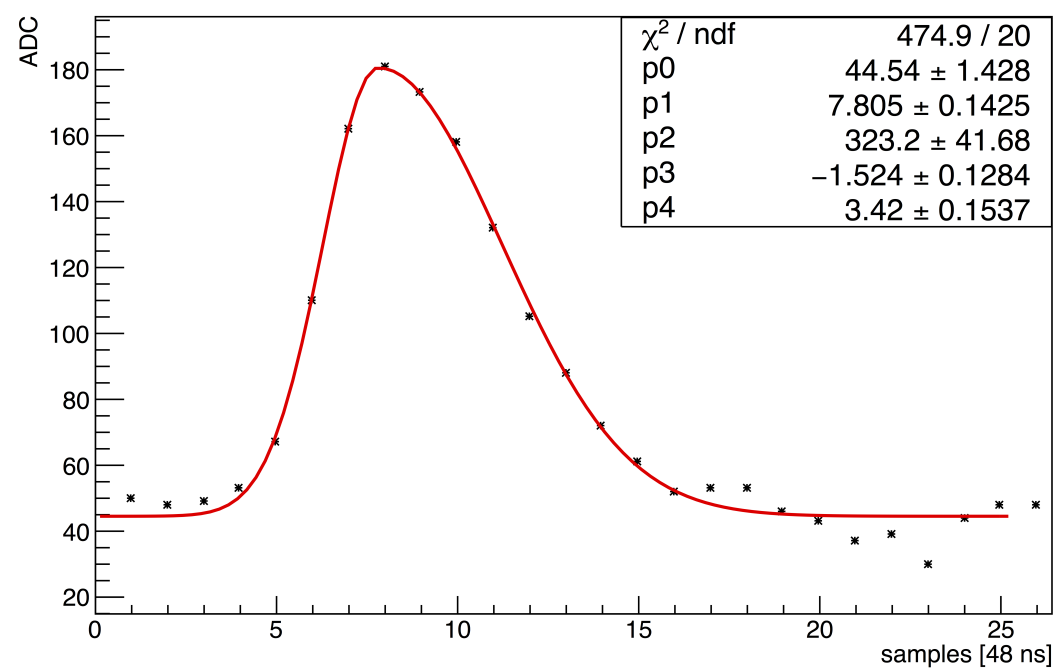

FIG. 33: Double Gaussian fit [Ch 9 - Evt 17]

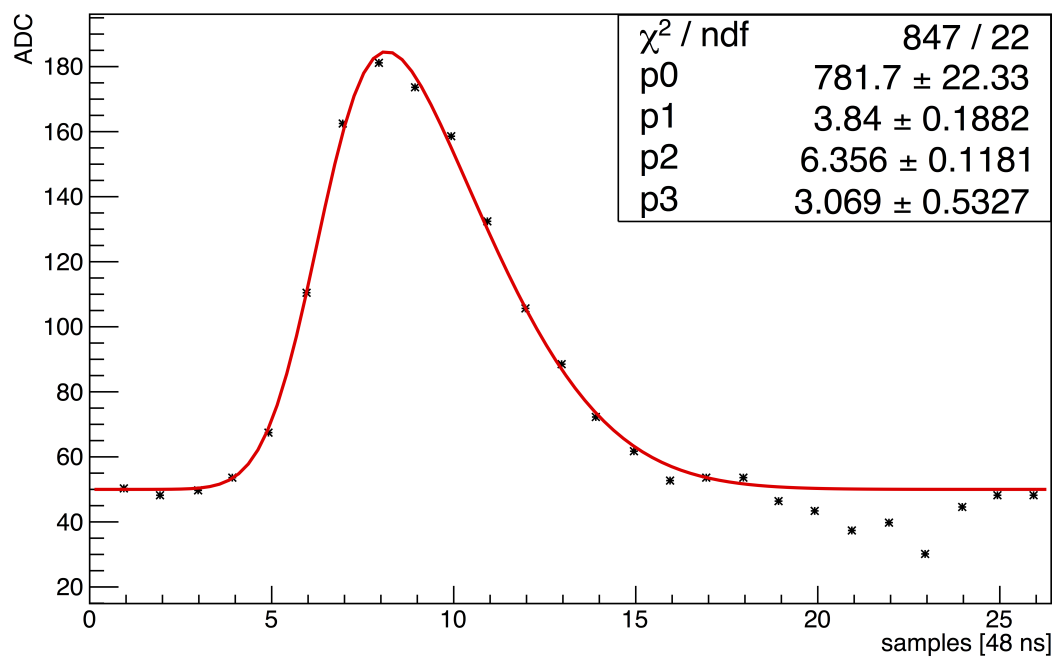

FIG. 34: Skewed Normal Distribution fit [Ch 9 - Evt 17] 


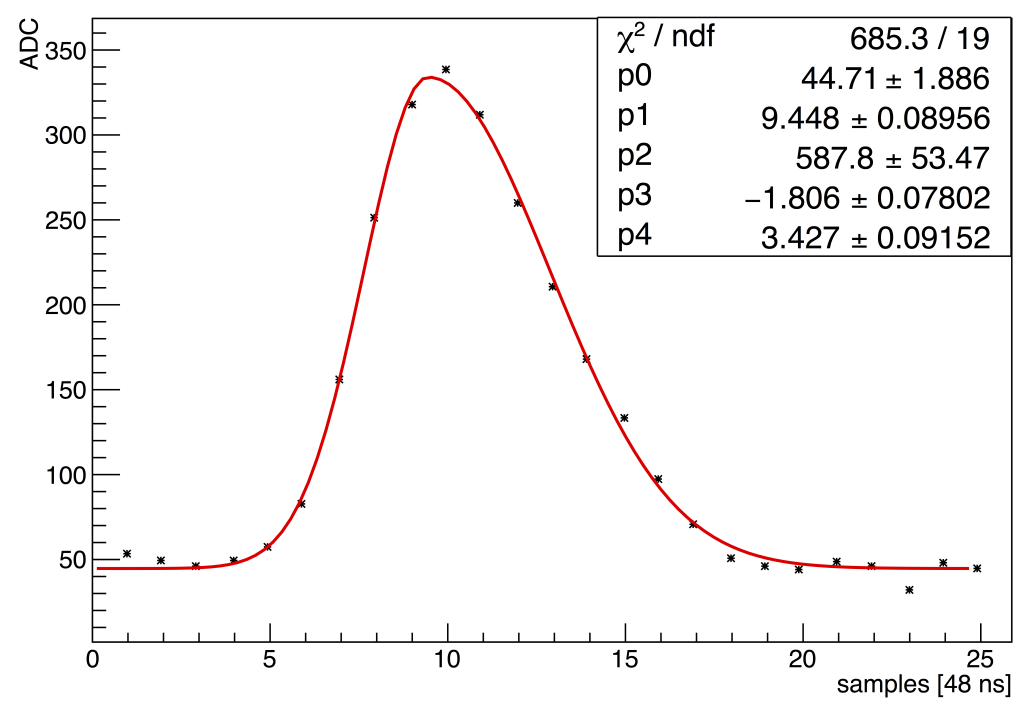

FIG. 35: Double Gaussian fit [Ch 35 - Evt 99]

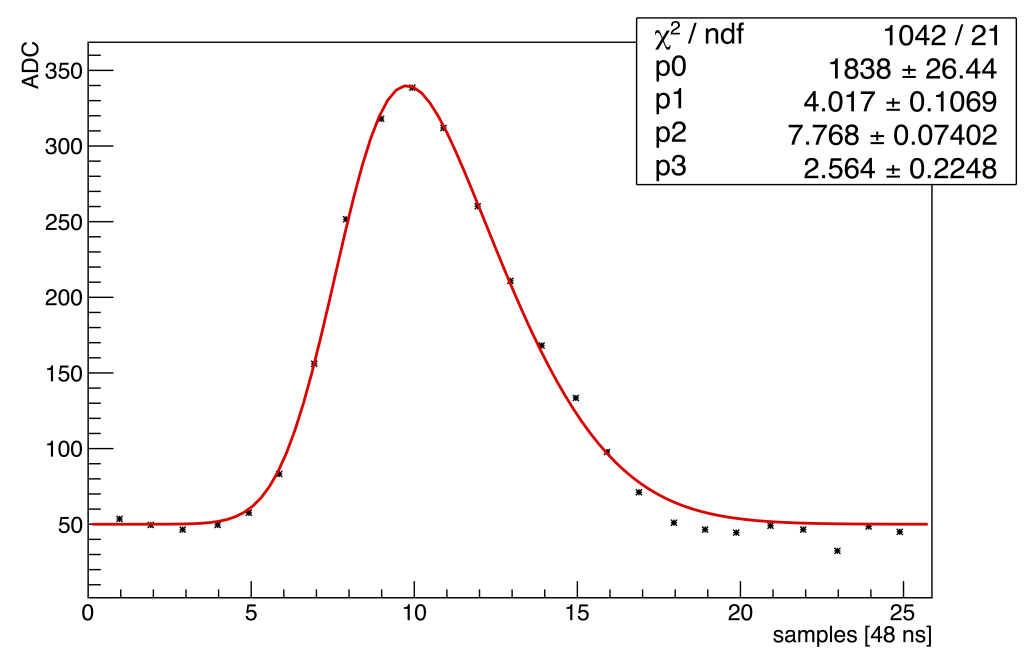

FIG. 36: Skewed Normal Distribution fit [Ch 35 - Evt 99]

According to the ratio $\chi^{2} / \mathrm{ndf}$, the double Gaussian is the best of the two functions to fit the signal. However, visually the skewed normal distribution looks to fit the points better. Other functions could fit the signal, and studies are on going to find the best one. 


\section{CHAPTER 5}

\section{CONCLUSION}

A test bench was successfully installed at Old Dominion University. The bench is composed of all the necessary equipments to use a RTPC similar to the one being designed for BONuS12 with a new readout electronics (DREAM-based). The results of the preliminary tests shows that the DREAM electronics works with the RTPC. ODU staff and students are beginning to have a good knowledge of the DREAM electronics and will be able to optimize its configuration for BONuS12 needs.

Finally, the first RTPC signals acquired with DREAM have been analyzed and fitted. While no final function to fit the signals was chosen, the first results are promising. 


\section{BIBLIOGRAPHY}

[1] R. Hofstadter and R. W. McAllister, Phys. Rev. 98, 217 (1955)

[2] M. N. Rosenbluth, Phys. Rev. 79, 615 (1950)

[3] W. Bartel et al., Phys. Lett. B28, 148 (1968)

[4] J. D. Bjorken, and E. A. Paschos, Phys. Rev. 185, 1975 (1969)

[5] M. Thomson, Modern Particle Physics (Cambridge University Press, 2013)

[6] C. C. degli Atti, L. P. Kaptari, and B. Z. Kopeliovich, Eur. Phys. J. A 19, 145 (2004)

[7] S. Tkachenko, et al. (CLAS Collaboration), Phys. Rev. C 89, 045206 (2014)

[8] A. Acardi, W. Melnitchouk, J. F. Owens, M. E. Christy, C. E. Keppel, et al., Phys. Rev. D 84, 014008 (2011)

[9] J. N. Marx, and D. R. Nygren, Physics Today 31, 10 (1978)

[10] F. Sauli, Nucl. Instrum. Meth. A 805, 2 (2016)

[11] H. Fenker, Nucl. Instrum. Meth. A 592, 273 (2008)

[12] R. Veenhof, Nucl. Instrum. Meth. A 419726 (1998)

[13] S. F. Biagi, Nucl. Instrum. Meth. A 421234 (1999)

[14] P. Baron, E. Delagnes, C. Flouzat, and F. Guilloux, User's Manual of DREAM, a Front End ASIC for CLAS12 detector, (2014)

[15] I. Mandjavidze, User's Manual of FEU, a frontend unit for Micromegas trackers, (2014)

[16] R. Brun, and F. Rademakers, Nucl. Instrum. Meth. A 38981 (1997) 


\section{VITA}

Mathieu Ehrhart

Department of Physics

Old Dominion University

Norfolk, VA 23529

\section{EDUCATION:}

- B.S. in Physics, Old Dominion University, Norfolk, VA, 2015

\section{EXPERIENCE AND RESEARCH:}

- 2015 - 2017: Research Assistant, Nuclear Physics Group, Old Dominion University, Norfolk VA

- 2017: Teaching Assistant, Department of Physics, Old Dominion University, Norfolk VA

- 2016: Research Aide, Argonne National Laboratory, Lemont, IL

- 2015: Summer Student, Organisation Européenne pour la Recherche Nucléaire (CERN), Switzerland/France

- 2014 - 2015: Undergraduate Research Assistant, Nuclear Physics Group, Old Dominion University, Norfolk VA

Typeset using $\mathrm{ATEX}_{\mathrm{E}}$ 